\title{
A second look at gauged supergravities from fluxes in M-theory
}

\author{
Jean-Pierre Derendinger and Adolfo Guarino \\ Albert Einstein Center for Fundamental Physics, \\ Institute for Theoretical Physics, Bern University, \\ Sidlerstraße 5, CH-3012 Bern, Switzerland \\ E-mail: derendinger@itp.unibe.ch, guarino@itp.unibe.ch
}

ABSTRACT: We investigate reductions of M-theory beyond twisted tori by allowing the presence of KK6 monopoles (KKO6-planes) compatible with $\mathcal{N}=4$ supersymmetry in four dimensions. The presence of KKO6-planes proves crucial to achieve full moduli stabilisation as they generate new universal moduli powers in the scalar potential. The resulting gauged supergravities turn out to be compatible with a weak $G_{2}$ holonomy at $\mathcal{N}=1$ as well as at some non-supersymmetric $\mathrm{AdS}_{4}$ vacua. The M-theory flux vacua we present here cannot be obtained from ordinary type IIA orientifold reductions including background fluxes, D6-branes (O6-planes) and/or KK5 (KKO5) sources. However, from a four-dimensional point of view, they still admit a description in terms of so-called non-geometric fluxes. In this sense we provide the M-theory interpretation for such non-geometric type IIA flux vacua.

KEYwords: Flux compactifications, Extended Supersymmetry, M-Theory, Supergravity Models

ARXiv EPRINT: 1406.6930 


\section{Contents}

1 Motivation 1

2 M-theory on $G_{2}$-manifolds with fluxes $\quad 4$

2.1 M-theory flux-induced superpotential 5

$\begin{array}{lll}2.2 & G_{2} \text {-structure of the M-theory reduction } & 7\end{array}$

$\begin{array}{llr}2.3 & \text { Interpretation as type IIA orientifolds } & 9\end{array}$

2.4 Cyclic symmetry and STU-models 11

3 Effective action and gauged supergravitites $\quad 12$

$\begin{array}{lll}3.1 & \text { Scherk-Schwarz reductions and BI } & 12\end{array}$

$\begin{array}{lll}3.2 & \text { Extended supersymmetry and gaugings } & 13\end{array}$

3.3 KK6 monopoles and $\mathcal{N}=8 \rightarrow \mathcal{N}=4$ breaking $\quad 15$

3.4 Universal IIA moduli, KK6 monopoles and scalar potential 16

4 Taxonomy of M-theory flux vacua $\quad 18$

$\begin{array}{ll}4.1 \text { Exploiting dualities in the effective theory } & 19\end{array}$

$\begin{array}{lll}4.2 & \text { Backgrounds without KK6 (KKO6) } & 20\end{array}$

4.3 Backgrounds with KK6 (KKO6) 21

4.3.1 Including only KK6 $(\mathrm{KKO} 6) \rightarrow \mathrm{D} 6_{\|}\left(\mathrm{O} 6_{\|}\right)$sources 21

4.3.2 Including only KK6 (KKO6) $\rightarrow$ KK6 $\|\left(\mathrm{KKO}_{\|}\right)$sources 22

4.3.3 Including both types of KK6 (KKO6) sources 23

4.4 Monopoles and duality orbits of $\mathcal{N}=4$ gaugings 24

4.5 Overview of M-theory backgrounds with monopoles 26

$\begin{array}{llr}5 & \text { Summary and final remarks } & 28\end{array}$

$\begin{array}{ll}\text { A Backgrounds with only } \mathrm{KK}_{\|}\left(\mathrm{KKO6}_{\|}\right) & 29\end{array}$

B Backgrounds with $\mathrm{KK} 6_{\|}\left(\mathrm{KKO6}_{\|}\right) \& \mathrm{D} 6_{\|}\left(\mathrm{O6} 6_{\|}\right)$

\section{Motivation}

How to get masses from extra dimensions [1] has captured the attention of theoretical physicists during the last thirty five years. How massless theories in higher dimensions lead to massive theories in lower dimensions remains at the core of the connection between strings or M-theory and the real world. One may think of two approaches. The first is the top-down approach where a higher-dimensional theory like strings or M-theory is selected and then a lower-dimensional effective model is derived from the choice of a compactification scheme. In this way the dynamics in lower dimensions follows from the reduction 
prescription. The higher-dimensional interpretation of such effective models is clear but, as a downside, one often engineers classes of compactifications which do not produce satisfactory physics. Alternatively, the bottom-up approach begins with an effective field theory (EFT) in lower dimensions selected using low-energy dynamical or phenomenological criteria. Only then can one try to relate such well-motivated models to more fundamental theories in higher dimensions like strings or M-theory. This may be feasible if some guiding principles are respected in the selection of the EFT.

A crucial ingredient in the construction of an EFT expected to describe classes of strings/M-theory compactifications is the number of preserved or broken supersymmetries. This is even more relevant than the space-time dimension since the existence of supercharges severely restricts the field content and the structure of the effective action in all dimensions. For the cases of 32 (maximal) and 16 (half-maximal) supercharges in four dimensions (4d), the guiding principle which governs the structure of the EFT is the embedding tensor (ET) formalism. This framework allows for a systematic exploration of $\mathcal{N}=8$ (maximal) [2] and $\mathcal{N}=4$ (half-maximal) [3] effective supergravity models — in the form of gauged supergravities - which, on the other hand, are of special interest due to their plausible realisation in higher dimensions as maximally supersymmetric and 1/2-BPS backgrounds.

However the identification between parameters in the embedding tensor formalism and quantities in a higher-dimensional theory turns out to be a subtle task and has occasionally led to some confusion in the literature. This has been for instance the case for the effective STU-models of ref. [4] arising from massive type IIA orientifold reductions including background fluxes, D6-branes and O6-planes. These were the first string constructions featuring full moduli stabilisation in a vacuum without requiring non-perturbative effects, such as Euclidean brane instantons or gaugino condensation, to stabilise the Kähler moduli [5]. In ref. [4] an $\mathcal{N}=1$ flux-induced superpotential $W_{\text {IIA }}$ was presented and the fluxes (couplings in $\left.W_{\text {IIA }}\right)$ were related to $\mathcal{N}=4$ gauging parameters, thus establishing a correspondence between flux compactifications and $(\mathcal{N}=1$ truncations of $) \mathcal{N}=4$ gauged supergravity in the context of type IIA orientifold reductions (16 supercharges). The string vacuum of ref. [4] was reconsidered in ref. [6] and found to actually require the presence of KK5 monopoles due to a relation of the form $[6,7]$

$$
\omega \omega \neq 0 \Rightarrow \text { Net charge of KK5 (KKO5) sources }
$$

involving the Scherk-Schwarz metric $\omega$-flux along the six-dimensional internal space $X_{6}$. This result indicated the necessity to extend the twisted tori picture of ref. [1], which demands $\omega \omega=0$ as a consistency relation. However, and only after the advent of the embedding tensor formalism, a thorough study of type IIA orientifold reductions [8] showed that $\omega \omega \neq 0$ violates the consistency conditions of $\mathcal{N}=4$ gauged supergravity [3]. As a consequence, the string vacuum of ref. [4] is not a solution of $\mathcal{N}=4$ gauged supergravity although it still is a perfectly acceptable solution of the $\mathcal{N}=1$ supergravity specified

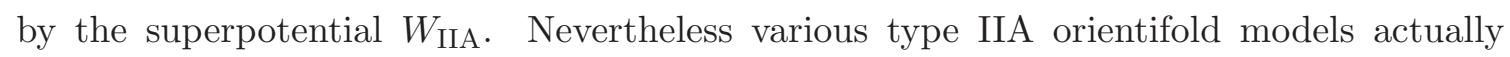
corresponding to $\mathcal{N}=4$ gauged supergravities, i.e. satisfying $\omega \omega=0$, have been worked out afterwards on the basis of the ET formalism $[8,9]$. In all the cases where full moduli stabilisation occurred, the massive version [10] of the type IIA theory was needed. 
Gauged supergravities related to M-theory reductions to four dimensions have been much less explored [11-14] than their type IIA relatives. ${ }^{1}$ Ref. [12] investigated in detail Scherk-Schwarz reductions on $G_{2}$-manifolds in the presence of background fluxes, derived an $\mathcal{N}=1$ flux-induced superpotential $W_{\text {M-theory }}$ and established the connection to the previous type IIA orientifold constructions by exploiting their underlying $\mathrm{SU}(3)$-structure. The resulting STU-models corresponded to $(\mathcal{N}=1$ truncations of $) \mathcal{N}=8$ gauged supergravities incompatible with full moduli stabilisation. Remarkably the authors identified a mismatch $^{2}$ between the $\mathcal{N}=1$ superpotentials of the M-theory models (32 supercharges) and of the type IIA orientifold models (16 supercharges) which can be summarised as

$$
W_{\mathrm{M} \text {-theory }}=\left.W_{\mathrm{IIA}}\right|_{a_{3}=0}-3 c_{3}^{\prime} T^{2}-3 d_{0} S T,
$$

where $a_{3}$ is the Romans mass ${ }^{3}$ and the flux parameters $\left(c_{3}^{\prime}, d_{0}\right)$ are metric $\omega$-fluxes in M-theory with no counterpart in the standard type IIA orientifold constructions. ${ }^{4}$ For this reason, they were set to zero in ref. [12] in order to have a neat $\mathrm{SU}(3) \subset \mathrm{G}_{2}$ embedding of the internal manifolds (6d vs 7d) underlying the type IIA orientifold and the M-theory reductions. In this work we will investigate several aspects of these genuine M-theory fluxes.

One of our main results is that full moduli stabilisation can be achieved in M-theory scenarios provided that the fluxes $\left(c_{3}^{\prime}, d_{0}\right)$ are activated. The minimally setup requires an $\mathcal{N}=8 \rightarrow \mathcal{N}=4$ breaking of supersymmetries (from 32 supercharges to 16 ) in the effective STU-models. Using the embedding tensor formalism as an organising principle for this we will derive a precise ET/flux dictionary in M-theory - we will show that the set of $\mathcal{N}=4$ consistency relations is compatible with a relaxation of the Scherk-Schwarz conditions $\omega \omega=0$ involving the metric $\omega$-flux in M-theory, in contrast to what happened in the type IIA case. Along the lines of ref. [6], we will introduce the corresponding KK6 monopoles entering the relation

$$
\omega \omega \neq 0 \Rightarrow \text { Net charge of KK6 (KKO6) sources },
$$

which now involves the Scherk-Schwarz metric $\omega$-flux along the seven-dimensional internal space $X_{7}$, and discuss their compatibility with preserving $\mathcal{N}=4$ supersymmetry in the effective action. The aim of this work is to extend the study of type IIA/M-theory reductions initiated in ref. [12] by exploiting the power of the embedding tensor formalism used to systematically analyse maximal and half-maximal gauged supergravitites in four dimensions.

The paper is organised as follows. In section 2 we review the reductions of M-theory on $G_{2}$-manifolds with fluxes [12] and their interpretation as type IIA orientifold constructions

\footnotetext{
${ }^{1}$ Consistent truncations of M-theory beyond the toroidal setup we discuss in this work have been discussed in refs [15-18].

${ }^{2}$ See also ref. [19].

${ }^{3}$ The IIA Romans mass parameter [10] is not generated upon (non-singular [20]) ordinary reductions of M-theory.

${ }^{4}$ They would correspond to non-geometric fluxes $[9,19,21]$ in a modern approach to type IIA flux compactifications.
} 
in order to introduce the effective STU-models considered in the rest of the paper. ${ }^{5}$ In section 3 we establish the precise correspondence between STU-models and (half-)maximal gauged supergravities in four dimensions. We present the flux/ET dictionary, discuss the interplay between supersymmetry and Scherk-Schwarz conditions as well as the relation to the absence/presence of KK6 monopoles and finally characterise the effective supergravity in terms of the universal moduli powers appearing in the scalar potential. In section 4 we exhaustively classify the structure of $4 \mathrm{~d}$ flux vacua by making a combined use of duality transformations in the STU-models and algebraic geometry techniques in order to solve the extremum conditions of the scalar potential and the consistency relations imposed by supersymmetry. A systematic analysis of the critical points identifying the required sources as well as the underlying $\mathcal{N}=4$ gauging is performed. We conclude with section 5 and present some relevant data associated to the M-theory flux vacua in the two appendices.

\section{M-theory on $G_{2}$-manifolds with fluxes}

Our starting point is the Scherk-Schwarz reduction of M-theory on $G_{2}$-manifolds with fluxes derived in ref. [12]. It is an orbifold reduction on $X_{7}=\frac{\mathbb{T}^{7}}{\mathbb{Z}_{2} \times \mathbb{Z}_{2} \times \mathbb{Z}_{2}}$ including $G_{(4)}$ and $G_{(7)}$ background fluxes for the $A_{(3)}$ and $A_{(6)}$ gauge potentials of $11 \mathrm{~d}$ supergravity, as well as a metric $\omega$-flux associated to a twist along the internal space $X_{7}$. We will re-derive the four dimensional effective theory of ref. [12] in order to establish the set of conventions we are using in this work.

Before introducing the twist, the $G_{2}$-holonomy of the orbifold is encoded in a $G_{2}$ invariant three-form and its $7 \mathrm{~d}$ dual four-form

$$
\begin{aligned}
\varphi_{G_{2}} & =d y^{127}+d y^{347}+d y^{567}+d y^{135}-d y^{146}-d y^{362}-d y^{524}, \\
\star_{7} \varphi_{G_{2}} & =d y^{3456}+d y^{1256}+d y^{1234}-d y^{2467}+d y^{2357}+d y^{4517}+d y^{6137},
\end{aligned}
$$

satisfying $\varphi_{G_{2}} \wedge \star_{7} \varphi_{G_{2}}=7 d y^{1234567}$. We have abbreviated $d y^{A B C} \equiv d y^{A} \wedge d y^{B} \wedge d y^{C}$ and $d y^{A B C D} \equiv d y^{A} \wedge d y^{B} \wedge d y^{C} \wedge d y^{D}$ with $A=1, \ldots, 7$ in the above expressions. The metric of the internal space is simply the flat metric of the ambient $\mathbb{T}^{7}$, i.e. $d s_{7}^{2}=\sum\left(\eta^{A}\right)^{2}$, where $\eta^{A}=R_{A} d y^{A}$ and $R_{A=1, \ldots, 7}$ denote the radii of the seven internal circles. We denote $\Phi_{(3)}\left(R_{A}\right)$ the deformed $\varphi_{G_{2}}$ with radii values $R_{A} \neq 1$, namely,

$$
\Phi_{(3)}=\eta^{127}+\eta^{347}+\eta^{567}+\eta^{135}-\eta^{146}-\eta^{362}-\eta^{524} .
$$

Consequently the internal component of the gauge potential $A_{(3)}$ has a similar expansion and both can be combined into a $G_{2}$ invariant complexified three-form

$$
\frac{1}{2}\left(A_{(3)}+i \Phi_{(3)}\right)=\sum_{A=1}^{7} T_{A}(x) \omega_{A}(y),
$$

\footnotetext{
${ }^{5}$ The STU-models we will discuss correspond to consistent $\mathrm{SO}(3)$ truncations of the $\frac{\mathrm{SL}(2) \times \mathrm{SO}(6,6)}{\mathrm{SO}(2) \times \mathrm{SO}(6) \times \mathrm{SO}(6)}$ coset space spanned by the scalar fields of half-maximal supergravity in four dimensions. The underlying group theory structure guarantees that we are actually solving the full set of equations of motion and not any truncated version thereof, even though we are setting most of the scalars to zero. As usual in supergravity theories (see ref. [22] for a recent discussion), the masses of the fields retained in the truncation are not necessarily the lightest ones and therefore the analysis of stability requires the knowledge of the full mass spectrum. We provide the complete spectrum for all vacua discussed in the paper in the appendices.
} 
where the $\omega_{A}(y)$ entering the above expansion are the seven basis elements of $H^{3}\left(X_{7}\right)$. The seven coefficients $T_{A}(x)$ represent moduli fields in the four-dimensional effective action.

After a twist is turned on by means of a metric flux, ${ }^{6}$ i.e. $\omega_{B C}{ }^{A} \neq 0$, the $G_{2}$-holonomy of the original orbifold is replaced by a $G_{2}$-structure. The set of left invariant twisted forms $\eta^{A}$ along the internal space then satisfy the Maurer-Cartan equations

$$
d \eta^{A}+\frac{1}{2} \omega_{B C}{ }^{A} \eta^{B} \wedge \eta^{C}=0
$$

and can be used to build the set $H^{p}\left(X_{7}\right)$ of cohomology classes of $X_{7}$.

The preserved $G_{2}$-structure ensures $\mathcal{N}=1$ supersymmetry in the reduced theory. The Kähler potential for the seven moduli fields $T_{A}$ in the expansion $(2.3)$ is given by $[12,23]$

$$
K=-\sum_{A=1}^{7} \log \left(-i\left(T_{A}-\bar{T}_{A}\right)\right),
$$

corresponding to a scalar manifold $\mathcal{M}_{\text {scalar }}=[\mathrm{SU}(1,1) / \mathrm{U}(1)]^{7}$. In addition a scalar potential also emerges upon reduction - see refs [23-25] for reductions with $G_{2}$-holonomy and refs $[11,12]$ for weak $G_{2}$-holonomy and cocalibrated $G_{2}$-structures -. This potential can be derived from the flux-induced superpotential [11, 12]

$$
W_{\mathrm{M} \text {-theory }}=\frac{1}{4} \int_{X_{7}} G_{(7)}+\frac{1}{4} \int_{X_{7}}\left(A_{(3)}+i \Phi_{(3)}\right) \wedge\left[G_{(4)}+\frac{1}{2} d\left(A_{(3)}+i \Phi_{(3)}\right)\right]
$$

using the standard $\mathcal{N}=1$ supergravity formula

$$
V=e^{K}\left[K^{A \bar{B}} D_{A} W D_{\bar{B}} \bar{W}-3 W \bar{W}\right],
$$

where $K^{A \bar{B}}$ is the inverse of the Kähler metric $K_{A \bar{B}}=\partial_{A} \partial_{\bar{B}} K$ and $D_{A} W=\partial_{A} W+$ $\left(\partial_{A} K\right) W$ is the Kähler derivative. The exterior derivative entering the last term in (2.6) corresponds to the twisted operator $d=\partial+\omega$ that incorporates the metric $\omega$-flux in the internal space $X_{7}$. The superpotential (2.6) consists of three pieces: the first piece is induced by $G_{(7)}$ an produces a constant term. The second piece is induced by $G_{(4)}$ and gives rise to linear couplings for the seven moduli. The third piece is induced by the metric $\omega$-flux and produces quadratic terms $T_{A} T_{B}$ (with $A \neq B$ ) in the superpotential.

\subsection{M-theory flux-induced superpotential}

Let us now derive the form of the M-theory superpotential (2.6) in the case of a reduction on $X_{7}=\mathbb{T}^{7} /\left(\mathbb{Z}_{2} \times \mathbb{Z}_{2} \times \mathbb{Z}_{2}\right)$ which has untwisted Betti numbers $b_{1}\left(X_{7}\right)=b_{2}\left(X_{7}\right)=0$ and $b_{3}\left(X_{7}\right)=7$. The geometry of the orbifold is encoded in its sets of invariant forms. Splitting the basis of left invariant twisted 1-forms as

$$
\eta^{A}=\left(\eta^{a}, \eta^{i}, \eta^{7}\right)
$$

\footnotetext{
${ }^{6}$ The $\omega$-metric flux $\omega_{B C}{ }^{A}=\omega_{[B C]}^{A}$ contains the 140' (traceless part) and 7 ' irrep's of SL(7) as can be seen from the tensor product $21^{\prime} \times \mathbf{7}=\mathbf{1 4 0} 0^{\prime}+\mathbf{7}^{\prime}$.
} 
with $a=1,3,5$ and $i=2,4,6$, then the seven basis elements of $H^{3}\left(X_{7}\right)$ are given by

$$
\begin{aligned}
& \omega_{1}=\eta^{12} \wedge \eta^{7}, \quad \omega_{2}=\eta^{34} \wedge \eta^{7}, \omega_{3}=\eta^{56} \wedge \eta^{7}, \\
& \alpha_{0}=\eta^{135}, \quad \beta^{1}=\eta^{146}, \quad \beta^{2}=\eta^{362}, \quad \beta^{3}=\eta^{524} \text {. }
\end{aligned}
$$

The complementary elements spanning $H^{4}\left(X_{7}\right)$ are then obtained by $7 \mathrm{~d}$ Hodge duality and read

$$
\begin{array}{lll}
\tilde{\omega}^{1}=\eta^{3456}, & \tilde{\omega}^{2}=\eta^{1256}, & \tilde{\omega}^{3}=\eta^{1234} \\
\beta^{0}=\eta^{246} \wedge \eta^{7}, & \alpha_{1}=\eta^{235} \wedge \eta^{7}, & \alpha_{2}=\eta^{451} \wedge \eta^{7}, \quad \alpha_{3}=\eta^{613} \wedge \eta^{7} .
\end{array}
$$

The cohomology basis then satisfies the orthogonality conditions

$$
\int_{X_{7}} \omega_{I} \wedge \tilde{\omega}^{J}=\mathcal{V}_{7} \delta_{I}^{J}, \quad \int_{X_{7}} \alpha_{0} \wedge \beta^{0}=-\mathcal{V}_{7}, \quad \int_{X_{7}} \beta^{I} \wedge \alpha_{J}=-\mathcal{V}_{7} \delta_{J}^{I},
$$

with $I, J=1,2,3$ and where the volume of $X_{7}$ is defined as $\mathcal{V}_{7}=\int_{X_{7}} \eta^{1234567}$.

Using the above set of invariant forms, it is possible to turn on background fluxes for $G_{(4)}$ and $G_{(7)}$ as well as for the metric $\omega$-flux. In terms of the elements in (2.10), the $G_{(4)}$ background flux can be expanded as

$$
\frac{1}{2} G_{(4)}=-\sum_{I} a_{1}{ }^{(I)} \tilde{\omega}^{I}+b_{0} \beta^{0}+\sum_{I} c_{0}^{(I)} \alpha_{I} .
$$

The expansion of the background for $G_{(7)}$ is simply

$$
\frac{1}{4} G_{(7)}=a_{0} \eta^{1234567} \text {. }
$$

In addition to the gauge fluxes (2.12) and (2.13), there are 21 metric $\omega$-fluxes compatible with the orbifold symmetries. The entire set of M-theory fluxes is summarised in table 1.

In terms of the basis elements (2.9), the expansion of the complex three-form in (2.3) can be rewritten as

$$
\frac{1}{2}\left(A_{(3)}+i \Phi_{(3)}\right)=\sum_{I} U_{I} \omega_{I}+S \alpha_{0}-\sum_{I} T_{I} \beta^{I},
$$

where $S, T_{I}$ and $U_{I}$ have the type IIA interpretation of dilaton, complex structure and Kähler moduli, respectively. ${ }^{7}$ Moreover we also find

$$
\frac{1}{2} d\left(A_{(3)}+i \Phi_{(3)}\right)=\sum_{I} P_{I} \tilde{\omega}^{I}+\beta^{0} \sum_{K}\left(d_{0}^{(K)} T_{K}-b_{1}^{(K)} U_{K}\right)+\sum_{I} Q^{I} \alpha_{I},
$$

where we have defined the quantities ${ }^{8}$

$$
\begin{array}{ll}
P_{I}=a_{2}^{(J)} U_{K}+a_{2}^{(K)} U_{J}+b_{1}^{(I)} S+\sum_{L} \mathcal{C}_{1}^{(I L)} T_{L} & (I \neq J \neq K), \\
Q^{I}=-c_{3}^{\prime(J)} T_{K}-c_{3}^{\prime(K)} T_{J}-d_{0}^{(I)} S+\sum_{L} U_{L} \mathcal{C}_{1}^{(L I)} & (I \neq J \neq K),
\end{array}
$$

\footnotetext{
${ }^{7}$ Notice the somehow unconventional names for the type IIA moduli fields. We have made this choice in order to exactly reproduce the generalised superpotential of ref. [9] derived in the context of type IIB compactifications and further connected to the embedding tensor framework for $\mathcal{N}=4$ supergravity.

${ }^{8}$ In the expressions $(2.16)$ the $I \neq J \neq K$ assignments have to be understood in a cyclic manner, namely $(I, J, K)=(1,2,3),(2,3,1),(3,1,2)$. For instance one has $P_{1}=a_{2}^{(2)} U_{3}+a_{2}^{(3)} U_{2}+b_{1}^{(1)} S+\sum_{L} \mathcal{C}_{1}^{(1 L)} T_{L}$ and similarly for the rest.
} 


\begin{tabular}{|c|c|c|}
\hline M-theory origin & Components & Fluxes \\
\hline$\omega_{b c}{ }^{a}$ & $\omega_{35}{ }^{1}, \omega_{51}{ }^{3}, \omega_{13}{ }^{5}$ & $\tilde{c}_{1}^{(1)}, \tilde{c}_{1}^{(2)}, \tilde{c}_{1}^{(3)}$ \\
\hline$\omega_{a j}{ }^{k}$ & $\omega_{14}{ }^{6}, \omega_{36}{ }^{2}, \omega_{52}{ }^{4}$ & $\hat{c}_{1}^{(1)}, \hat{c}_{1}^{(2)}, \hat{c}_{1}^{(3)}$ \\
\hline$\omega_{k a}{ }^{j}$ & $\omega_{61}{ }^{4}, \omega_{23}{ }^{6}, \omega_{45}{ }^{2}$ & $\check{c}_{1}^{(1)}, \check{c}_{1}^{(2)}, \check{c}_{1}^{(3)}$ \\
\hline$\omega_{j k}{ }^{a}$ & $\omega_{46}{ }^{1}, \omega_{62}{ }^{3}, \omega_{24}{ }^{5}$ & $b_{1}^{(1)}, b_{1}^{(2)}, b_{1}^{(3)}$ \\
\hline$-\omega_{a i}{ }^{7}$ & $-\omega_{12}{ }^{7},-\omega_{34}{ }^{7},-\omega_{56}{ }^{7}$ & $a_{2}^{(1)}, a_{2}^{(2)}, a_{2}^{(3)}$ \\
\hline$-\omega_{7 i}{ }^{a}$ & $-\omega_{72}{ }^{1},-\omega_{74}{ }^{3},-\omega_{76}{ }^{5}$ & $d_{0}^{(1)}, d_{0}^{(2)}, d_{0}^{(3)}$ \\
\hline$-\omega_{a 7}{ }^{i}$ & $-\omega_{17}{ }^{2},-\omega_{37}{ }^{4},-\omega_{57}{ }^{6}$ & $c_{3}^{(1)}, c_{3}^{(2)}, c_{3}^{(3)}$ \\
\hline$-\frac{1}{2} G_{a i b j}$ & $-\frac{1}{2} G_{3456},-\frac{1}{2} G_{1256},-\frac{1}{2} G_{1234}$ & $a_{1}^{(1)}, a_{1}^{(2)}, a_{1}^{(3)}$ \\
\hline$\frac{1}{2} G_{i j k 7}$ & $\frac{1}{2} G_{2467}$ & $b_{0}$ \\
\hline$\frac{1}{2} G_{i b c 7}$ & $\frac{1}{2} G_{2357}, \frac{1}{2} G_{4517}, \frac{1}{2} G_{6137}$ & $c_{0}^{(1)}, c_{0}^{(2)}, c_{0}^{(3)}$ \\
\hline$\frac{1}{4} G_{a i b j c k 7}$ & $\frac{1}{4} G_{1234567}$ & $a_{0}$ \\
\hline
\end{tabular}

Table 1. Metric and gauge fluxes entering the M-theory superpotential.

and where $\mathcal{C}_{1}$ is the flux matrix introduced in ref. [26]

$$
\mathcal{C}_{1}^{(I J)}=\left(\begin{array}{ccc}
-\tilde{c}_{1}^{(1)} & \check{c}_{1}^{(3)} & \hat{c}_{1}^{(2)} \\
\hat{c}_{1}^{(3)} & -\tilde{c}_{1}^{(2)} & \check{c}_{1}^{(1)} \\
\check{c}_{1}^{(2)} & \hat{c}_{1}^{(1)} & -\tilde{c}_{1}^{(3)}
\end{array}\right) .
$$

By plugging (2.12)-(2.15) into the flux-induced superpotential (2.6) and using the orthogonality conditions (2.11), one finds the M-theory superpotential

$$
\begin{aligned}
W_{\text {M-theory }}= & a_{0}-b_{0} S+\sum_{K=1}^{3} c_{0}^{(K)} T_{K}-\sum_{K=1}^{3} a_{1}^{(K)} U_{K} \\
& +\sum_{K=1}^{3} a_{2}^{(K)} \frac{U_{1} U_{2} U_{3}}{U_{K}}+\sum_{I, J=1}^{3} U_{I} \mathcal{C}_{1}^{(I J)} T_{J}+S \sum_{K=1}^{3} b_{1}^{(K)} U_{K} \\
& -\sum_{K=1}^{3} c_{3}^{\prime(K)} \frac{T_{1} T_{2} T_{3}}{T_{K}}-S \sum_{K=1}^{3} d_{0}^{(K)} T_{K} .
\end{aligned}
$$

With this we conclude the re-derivation of the effective supergravities coming from twisted reductions of M-theory on an $X_{7}=\mathbb{T}^{7} /\left(\mathbb{Z}_{2} \times \mathbb{Z}_{2} \times \mathbb{Z}_{2}\right)$ orbifold with fluxes and set up the scenario we will analyse later.

\section{$2.2 G_{2}$-structure of the M-theory reduction}

The geometry of the twisted $X_{7}=\mathbb{T}^{7} /\left(\mathbb{Z}_{2} \times \mathbb{Z}_{2} \times \mathbb{Z}_{2}\right)$ orbifold we are considering determines the set of $G_{2}$-structure relations

$$
\begin{aligned}
d \Phi_{(3)} & =\widetilde{W}_{1} \star_{7} \Phi_{(3)}+2 \widetilde{W}_{27}, \\
d \star_{7} \Phi_{(3)} & =0,
\end{aligned}
$$


thus corresponding to a cocalibrated $\left(\widetilde{W}_{7}=\widetilde{W}_{14}=0\right) G_{2}$-structure $[12,27]$. Let us explicitly compute the torsion classes $\widetilde{W}_{1}$ and $\widetilde{W}_{27}$ sitting respectively in the $\mathbf{1}$ and $\mathbf{2 7}$ irrep's of $\mathrm{G}_{2}$ in the particular case of $A_{(3)}=0$ and $R_{A}=1$ for the seven radii in $X_{7}$. This requires to evaluate the expression (2.14) at the point $S=T_{I}=U_{I}=i$ so that the twisted versions of the $G_{2}$ invariant forms in (2.1) are recovered. These are

$$
\frac{1}{2} \Phi_{(3)}=\sum_{I=1}^{3} \omega_{I}+\alpha_{0}-\sum_{I=1}^{3} \beta^{I}, \quad \frac{1}{2} \star_{7} \Phi_{(3)}=\sum_{I=1}^{3} \tilde{\omega}_{I}-\beta^{0}+\sum_{I=1}^{3} \alpha_{I},
$$

with the non-standard normalisation $\frac{1}{7} \int_{X_{7}} \Phi_{(3)} \wedge \star_{7} \Phi_{(3)}=4 \mathcal{V}_{7}$. After some algebra we obtain a one-parameter family - the non-trivial condition in (2.19) is linear — of torsion classes satisfying (2.19). It is given by

$$
\widetilde{W}_{1}=(1+\kappa) W_{1} \quad \text { and } \quad 2 \widetilde{W}_{27}=2 W_{27}-\kappa W_{1} \star_{7} \Phi_{(3)},
$$

where we have introduced the flux-dependent quantities

$$
\begin{aligned}
W_{1} & =\sum_{L} a_{2}^{(L)}+\sum_{L} b_{1}^{(L)}+\sum_{I J} \mathcal{C}_{1}^{(I J)}-\sum_{L} d_{0}^{(L)}-\sum_{L} c_{3}^{(L)}, \\
W_{27} & =\sum_{I} A_{I} \tilde{\omega}^{I}+B \beta^{0}+\sum_{I} C_{I} \alpha_{I} .
\end{aligned}
$$

The coefficients in the expansion of $W_{27}$ also depend on the flux parameters and $\operatorname{read}^{9}$

$$
\begin{aligned}
A_{I} & =-a_{2}^{(I)}-b_{1}^{(J)}-b_{1}^{(K)}-\sum_{L}\left(\mathcal{C}_{1}^{(J L)}+\mathcal{C}_{1}^{(K L)}\right)+\sum_{L}\left(d_{0}^{(L)}+c_{3}^{(L)}\right)(I \neq J \neq K) \\
B & =\sum_{L} a_{2}^{(L)}+\sum_{I J} \mathcal{C}_{1}^{(I J)}-\sum_{L} c_{3}^{(L)} \\
C_{I} & =c_{3}^{\prime(I)}+d_{0}^{(J)}+d_{0}^{(K)}-\sum_{L}\left(\mathcal{C}_{1}^{(L J)}+\mathcal{C}_{1}^{(L K)}\right)-\sum_{L}\left(a_{2}^{(L)}+b_{1}^{(L)}\right)(I \neq J \neq K) .
\end{aligned}
$$

In order to recover the standard $G_{2}$ relations for the properly normalised $\Phi_{(3)}$ and $\star_{7} \Phi_{(3)}$ forms $[28,29]$

$$
\left.\widetilde{W}_{1}=\frac{1}{7}\left(\frac{1}{2} d \Phi_{(3)}\right)\right\lrcorner\left(\frac{1}{2} \star_{7} \Phi_{(3)}\right) \quad \text { and } \quad \widetilde{W}_{27}=\left(\frac{1}{2} d \Phi_{(3)}\right)-\widetilde{W}_{1}\left(\frac{1}{2} \star_{7} \Phi_{(3)}\right),
$$

one must set the parameter $\kappa=-5 / 7$ in (2.21). Up to an overall $\frac{1}{16}$ factor coming from the normalisation of (2.6), this is consistent with the relation [29] between the potential energy induced by the metric $\omega$-flux and the Ricci scalar of $X_{7}$

$$
V_{\omega}=-\frac{1}{16} \operatorname{Ricci}_{X_{7}}=-\frac{1}{16}\left(\frac{21}{8}\left|\widetilde{W}_{1}\right|^{2}-\frac{1}{2}\left|\widetilde{W}_{27}\right|^{2}\right) .
$$

Generic M-theory flux vacua will activate the two torsion classes $\widetilde{W}_{1}$ and $\widetilde{W}_{27}$ thus specifying a cocalibrated $G_{2}$-structure. However under certain circumstances - for instance at $\mathcal{N}=1$ supersymmetric $\mathrm{AdS}_{4}$ solutions [12] — one might have $\widetilde{W}_{27}=0$ determining a weak $G_{2}$-holonomy or even $\widetilde{W}_{1}=\widetilde{W}_{27}=0$ restoring a $G_{2}$-holonomy. We will investigate this issue for the set of M-theory flux vacua we will obtain in section 4.

\footnotetext{
${ }^{9}$ As in (2.16), the $I \neq J \neq K$ assignments are understood in a cyclic manner also in (2.23). This time one has $A_{1}=-a_{2}^{(1)}-b_{1}^{(2)}-b_{1}^{(3)}-\sum_{L}\left(\mathcal{C}_{1}^{(2 L)}+\mathcal{C}_{1}^{(3 L)}\right)+\sum_{L}\left(d_{0}^{(L)}+c_{3}^{(L)}\right)$ and similarly for the others.
} 


\subsection{Interpretation as type IIA orientifolds}

Massless type IIA supergravity can be obtained from reduction of M-theory along the 11th direction. Schematically, this amounts to the splitting

$$
X_{7}=\frac{\mathbb{T}^{7}}{\mathbb{Z}_{2} \times \mathbb{Z}_{2} \times \mathbb{Z}_{2}} \longrightarrow \frac{\mathbb{T}^{6}}{\mathbb{Z}_{2} \times \mathbb{Z}_{2}} \times \eta^{7}=X_{6} \times \eta^{7}
$$

with $\eta^{m=1, \ldots, 6}$ being associated to $X_{6}$, additionally endowed with an extra $\mathbb{Z}_{2}$ "orientifold" involution reflecting the coordinates $\eta^{i} \rightarrow-\eta^{i}$ and $\eta^{7} \rightarrow-\eta^{7}$. This is compatible with the following invariant forms. From the forms in (2.9) and (2.10), one reads off the elements spanning $H^{2}\left(X_{6}\right)$

$$
\omega_{1}=\eta^{12}, \quad \omega_{2}=\eta^{34}, \quad \omega_{3}=\eta^{56},
$$

as well as those spanning $H^{4}\left(X_{6}\right)$

$$
\tilde{\omega}^{1}=\eta^{3456}, \quad \tilde{\omega}^{2}=\eta^{1256}, \quad \tilde{\omega}^{3}=\eta^{1234} .
$$

Similarly, the elements spanning $H^{3}\left(X_{6}\right)$ are given by

$$
\begin{array}{llll}
\alpha_{0}=\eta^{135}, & \beta^{1}=\eta^{146}, & \beta^{2}=\eta^{362}, & \beta^{3}=\eta^{524} \\
\beta^{0}=\eta^{246}, & \alpha_{1}=\eta^{235}, & \alpha_{2}=\eta^{451}, & \alpha_{3}=\eta^{613} .
\end{array}
$$

The volume of $X_{6}$ is then defined as $\mathcal{V}_{6}=\int_{X_{6}} \eta^{123456}$ and the orthogonality conditions in (2.11) tranlates into

$$
\int_{X_{6}} \omega_{I} \wedge \tilde{\omega}^{J}=\mathcal{V}_{6} \delta_{I}^{J}, \quad \int_{X_{6}} \alpha_{0} \wedge \beta^{0}=-\mathcal{V}_{6}, \quad \int_{X_{6}} \beta^{I} \wedge \alpha_{J}=-\mathcal{V}_{6} \delta_{J}^{I} .
$$

Analogously to the M-theory case, background fluxes for all the type IIA gauge potentials can be turned on together with a type IIA metric $\omega$-flux ${ }^{10}$ in the internal space $X_{6}$. In terms of the cohomology basis (2.27)-(2.29), the R-R background fluxes can be expanded as

$$
F_{(6)}=a_{0} \eta^{123456}, \quad F_{(4)}=-a_{1}^{(I)} \tilde{\omega}^{I}, \quad F_{(2)}=a_{2}^{(I)} \omega_{I}, \quad F_{(0)}=-a_{3}
$$

whereas the expansion of the NS-NS flux can be taken as

$$
H_{(3)}=b_{0} \beta^{0}+c_{0}^{(I)} \alpha_{I} \text {. }
$$

Importantly, the $F_{(0)}=-a_{3}$ flux parameter in (2.31) corresponds to the Romans mass in massive type IIA supergravity [10] and does not directly descend from M-theory. The full set of type IIA fluxes including also metric fluxes is summarised in table 2 .

\footnotetext{
${ }^{10}$ The type IIA metric $\omega$-flux $\omega_{n p}{ }^{m}=\omega_{[n p]}{ }^{m}$ contains the 84' (traceless part) and 6' irrep's of SL(6) as

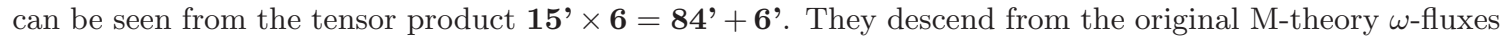
by virtue of the $\mathrm{SL}(7) \supset \mathrm{SL}(6)$ decompositions $\mathbf{1 4 0} \mathbf{\prime}^{\prime} \rightarrow \mathbf{8 4}^{\prime}+\mathbf{1 5}^{\prime}+\mathbf{3 5}+\mathbf{6}^{\prime}$ and $\mathbf{7}^{\prime} \boldsymbol{\rightarrow} \mathbf{6}^{\prime}+\mathbf{1}$ which can be equivalently viewed as $\omega_{B C}{ }^{A} \rightarrow \omega_{n p}{ }^{m} \oplus \omega_{n p}{ }^{7} \oplus \omega_{7 p^{m}}{ }^{m} \omega_{7 p}{ }^{7}$ and $\omega_{B C}{ }^{C} \rightarrow \omega_{n C}{ }^{C} \oplus \omega_{7 C}{ }^{C}$. The orbifold symmetries reduce the number of IIA metric fluxes $\omega_{n p}{ }^{m}$ to 12 .
} 


\begin{tabular}{|c|c|c|}
\hline Type IIA origin & Components & Fluxes \\
\hline$\omega_{b c}^{a}$ & $\omega_{35}{ }^{1}, \omega_{51}{ }^{3}, \omega_{13}{ }^{5}$ & $\tilde{c}_{1}^{(1)}, \tilde{c}_{1}^{(2)}, \tilde{c}_{1}^{(3)}$ \\
\hline$\omega_{a j}^{k}$ & $\omega_{14}^{6}, \omega_{36}{ }^{2}, \omega_{52}{ }^{4}$ & $\hat{c}_{1}^{(1)}, \hat{c}_{1}^{(2)}, \hat{c}_{1}^{(3)}$ \\
\hline$\omega_{k a^{j}}$ & $\omega_{61}^{4}, \omega_{23}{ }^{6}, \omega_{45}{ }^{2}$ & $\check{c}_{1}^{(1)}, \check{c}_{1}^{(2)}, \check{c}_{1}^{(3)}$ \\
\hline$\omega_{j k}^{a}$ & $\omega_{46}{ }^{1}, \omega_{62}{ }^{3}, \omega_{24}^{5}$ & $b_{1}^{(1)}, b_{1}^{(2)}, b_{1}^{(3)}$ \\
\hline$F_{a i}$ & $F_{12}, F_{34}, F_{56}$ & $a_{2}^{(1)}, a_{2}^{(2)}, a_{2}^{(3)}$ \\
\hline \multicolumn{2}{|c|}{ non-geometric } & $d_{0}^{(1)}, d_{0}^{(2)}, d_{0}^{(3)}$ \\
\hline \multicolumn{2}{|c|}{ non-geometric } & $c_{3}^{\prime(1)}, c_{3}^{\prime(2)}, c_{3}^{\prime(3)}$ \\
\hline$-F_{a i b j}$ & $-F_{3456},-F_{1256},-F_{1234}$ & $a_{1}^{(1)}, a_{1}^{(2)}, a_{1}^{(3)}$ \\
\hline$H_{i j k}$ & $H_{246}$ & $b_{0}$ \\
\hline$H_{i b c}$ & $H_{235}, H_{451}, H_{613}$ & $c_{0}^{(1)}, c_{0}^{(2)}, c_{0}^{(3)}$ \\
\hline$F_{\text {aibjck }}$ & $F_{123456}$ & $a_{0}$ \\
\hline$-F_{(0)}$ & (Romans mass) & $a_{3}$ \\
\hline
\end{tabular}

Table 2. Metric, gauge and non-geometric fluxes entering the type IIA superpotential.

In type IIA orientifold compactifications including O6-planes and D6-branes, the fluxinduced superpotential takes the form $[4,30]$

$$
W_{\mathrm{IIA}}=\int_{X_{6}} e^{J_{c}} \wedge F+\int_{X_{6}} \Omega_{c} \wedge\left(H_{(3)}+d J_{c}\right),
$$

where the (complexified) Kähler two-form $J_{c}$ and the holomorphic three-form $\Omega_{c}$ can be read off from (2.14) by requiring $\frac{1}{2}\left(A_{(3)}+i \Phi_{(3)}\right)=J_{c} \wedge \eta^{7}+\Omega_{c}$. This is

$$
J_{c}=\sum_{I} U_{I} \omega_{I} \quad \text { and } \quad \Omega_{c}=S \alpha_{0}-\sum_{I} T_{I} \beta^{I} .
$$

Using the type IIA metric $\omega$-fluxes in $X_{6}$ displayed in table 2 one finds

$$
d J_{c}=-\beta^{0} \sum_{K} b_{1}^{(K)} U_{K}+\alpha_{I} \sum_{L} U_{L} \mathcal{C}_{1}^{(L I)},
$$

and an explicit computation of the superpotential (2.33) yields

$$
\begin{aligned}
W_{\mathrm{IIA}}= & a_{0}-b_{0} S+\sum_{K=1}^{3} c_{0}^{(K)} T_{K}-\sum_{K=1}^{3} a_{1}^{(K)} U_{K} \\
& +\sum_{K=1}^{3} a_{2}^{(K)} \frac{U_{1} U_{2} U_{3}}{U_{K}}+\sum_{I, J=1}^{3} U_{I} \mathcal{C}_{1}^{(I J)} T_{J}+S \sum_{K=1}^{3} b_{1}^{(K)} U_{K}-a_{3} U_{1} U_{2} U_{3} .
\end{aligned}
$$

As noticed in refs $[12,19]$, the ordinary type IIA orientifold reductions including gauge plus metric fluxes miss the $c_{3}^{\prime(I)}$ and $d_{0}^{(I)}$ fluxes with respect to the ordinary M-theory 
construction of the previous sections. However, they gain the Romans mass parameter $a_{3}$ and the corresponding cubic coupling in the IIA superpotential (2.36). As a consequence the M-theory superpotential (2.18) can be viewed as a massless $\left(a_{3}=0\right)$ but generalised type IIA superpotential including the non-geometric fluxes $c_{3}^{\prime(I)}$ and $d_{0}^{(I)}$ which induce the last two terms in (2.18). The situation can be described as follows

$$
W_{\mathrm{M} \text {-theory }}=W_{\mathrm{IIA}}^{\left(a_{3}=0\right)}+W_{\text {non-geom }}=W_{\mathrm{IIA}}^{\left(a_{3}=0\right)}-\sum_{K=1}^{3} c_{3}^{\prime(K)} \frac{T_{1} T_{2} T_{3}}{T_{K}}-S \sum_{K=1}^{3} d_{0}^{(K)} T_{K} .
$$

We will elaborate more on the consequences of turning on these type IIA non-geometric fluxes $c_{3}^{(I)}$ and $d_{0}^{(I)}$ as well as on the interpretation of the corresponding flux-induced vacua as backgrounds containing KK monopoles, thus going beyond twisted tori as suggested in ref. [12] (see discussion in section 5.2 therein). Our approach here will be completely fourdimensional as we will be using the effective theory of $\mathcal{N}=4$ gauged supergravity [3] as the theoretical framework in which to describe the backgrounds.

\subsection{Cyclic symmetry and STU-models}

In order to simplify the setup as much as possible we will further restrict to the isotropic scenario in which a cyclic $\mathrm{SO}(3)$ symmetry $I \rightarrow J \rightarrow K$ is imposed [4]. This simplification is compatible with an Ansatz

$$
T_{1}=T_{2}=T_{3} \equiv T \quad \text { and } \quad U_{1}=U_{2}=U_{3} \equiv U
$$

for the four-dimensional moduli fields. The Kähler potential in (2.5) then reduces to the isotropic form

$$
K^{(\text {iso })}=-\log (-i(S-\bar{S}))-3 \log (-i(T-\bar{T}))-3 \log (-i(U-\bar{U}))
$$

which corresponds to a $\mathcal{M}_{\text {scalar }}=[\mathrm{SU}(1,1) / \mathrm{U}(1)]_{S} \times[\mathrm{SU}(1,1) / \mathrm{U}(1)]_{T} \times[\mathrm{SU}(1,1) / \mathrm{U}(1)]_{U}$ manifold described by the moduli fields of the so-called STU-model. This simplification is also consistent with an isotropic flux Ansatz of the form

$$
\tilde{c}_{1}^{(I)}=\tilde{c}_{1}, \quad \hat{c}_{1}^{(I)}=\check{c}_{1}^{(I)}=c_{1}, \quad b_{1}^{(I)}=b_{1}, \quad a_{2}^{(I)}=a_{2}, \quad d_{0}^{(I)}=d_{0}, \quad c_{3}^{\prime(I)}=c_{3}^{\prime}
$$

for the M-theory metric $\omega$-fluxes in table 1 and similarly for the gauge fluxes

$$
a_{1}^{(I)}=a_{1}, \quad c_{0}^{(I)}=c_{0} .
$$

The above content of fields and fluxes has been shown to be part of the $\mathrm{SO}(3)$ invariant sector of the maximal and half-maximal supergravities in four dimensions, the latter being coupled to six vector multiplets $[9,31]$. We will exploit this fact later on in the paper to investigate the effect of introducing M-theory monopoles in the compactification scheme.

In the isotropic limit, the expression (2.18) of the M-theory flux-induced superpotential takes the form

$$
\begin{aligned}
W_{\text {M-theory }}^{(\text {iso })}= & a_{0}-b_{0} S+3 c_{0} T-3 a_{1} U+3 a_{2} U^{2}+3\left(2 c_{1}-\tilde{c}_{1}\right) U T+3 b_{1} S U \\
& -3 c_{3}^{\prime} T^{2}-3 d_{0} S T,
\end{aligned}
$$


whereas the type IIA superpotential in (2.36) reduces to [4]

$$
W_{\mathrm{IIA}}^{\text {(iso) }}=a_{0}-b_{0} S+3 c_{0} T-3 a_{1} U+3 a_{2} U^{2}+3\left(2 c_{1}-\tilde{c}_{1}\right) U T+3 b_{1} S U-a_{3} U^{3} .
$$

These are the M-theory and type IIA superpotentials we will consider during the rest of the paper. Notice that the relation (2.37) still holds in its isotropic version

$$
W_{\mathrm{M} \text {-theory }}^{(\text {iso })}=W_{\mathrm{IIA}}^{(\mathrm{iso})\left(a_{3}=0\right)}+W_{\text {non-geom }}^{(\mathrm{iso})}=W_{\mathrm{IIA}}^{(\mathrm{iso})\left(a_{3}=0\right)}-3 c_{3}^{\prime} T^{2}-3 d_{0} S T,
$$

making the connection between M-theory and type IIA effective STU-models manifest.

The simplifications (2.40) and (2.41) on the fluxes also translate into simpler torsion classes $\widetilde{W}_{1}$ and $\widetilde{W}_{27}$ specifying the isotropic $G_{2}$-structure. The expressions $(2.22)$ and (2.23) simplify to

$$
\begin{aligned}
W_{1} & =3 a_{2}+3 b_{1}+3\left(2 c_{1}-\tilde{c}_{1}\right)-3 d_{0}-3 c_{3}^{\prime}, \\
W_{27} & =A \sum_{I} \tilde{\omega}^{I}+B \beta^{0}+C \sum_{I} \alpha_{I},
\end{aligned}
$$

with the flux-dependent coefficients in $W_{27}$ given by

$$
\begin{aligned}
& A=-a_{2}-2 b_{1}-2\left(2 c_{1}-\tilde{c}_{1}\right)+3 d_{0}+3 c_{3}^{\prime}, \\
& B=3 a_{2}+3\left(2 c_{1}-\tilde{c}_{1}\right)-3 c_{3}^{\prime}, \\
& C=c_{3}^{\prime}+2 d_{0}-2\left(2 c_{1}-\tilde{c}_{1}\right)-3 a_{2}-3 b_{1} .
\end{aligned}
$$

Constraining the torsion classes, e.g. demanding $\widetilde{W}_{27}=0$ to have weak $G_{2}$-holonomy, imposes linear relations on the background fluxes that simplify the resulting STU-models.

\section{$3 \quad$ Effective action and gauged supergravitites}

In this section we investigate the connection between the consistency conditions in ScherkSchwarz reductions of M-theory (top-down) and the consistency conditions in effective $\mathcal{N}=4$ and $\mathcal{N}=8$ gauged supergravities (bottom-up). We will link such conditions to the absence/presence of KK6 monopoles in the M-theory background and characterise the resulting scalar potential in the effective supergravity action.

\subsection{Scherk-Schwarz reductions and BI}

The M-theory fluxes are restricted by a set of quadratic constraints coming from the consistency of the reduction down to four dimensions $[1,12,32,33]$. In an ordinary ScherkSchwarz reduction of M-theory these are

$$
\omega_{[A B}^{F} \omega_{C] F}^{D}=0 \quad \text { and } \quad \omega_{[A B}^{F} G_{C D E] F}=0
$$

coming respectively from the nilpotency $\left(d^{2}=0\right)$ of the twisted derivative operator $d=\partial+\omega$ as well as from the twisted Bianchi identity (BI) $d G_{(4)}=0$ along the internal space $X_{7}$. Moreover the symmetries of the $X_{7}=\mathbb{T}^{7} /\left(\mathbb{Z}_{2} \times \mathbb{Z}_{2} \times \mathbb{Z}_{2}\right)$ orbifold guarantees $\omega_{A B}{ }^{A}=0$ 
(compact $X_{7}$ with no boundary), thus implying a well-defined Lagrangian upon reduction [1].

The first quadratic constraint in (3.1) gives rise to a set of $6+6+3+1+3+6+3=28$ conditions of the form ${ }^{11}$

$$
\begin{aligned}
& \text { i) } \omega_{[a i}{ }^{D} \omega_{c] D}{ }^{k}=0 \rightarrow-a_{2}^{(I)} c_{3}^{c^{(J)}}+\mathcal{C}_{1}^{(K K)} \mathcal{C}_{1}^{(J I)}+\mathcal{C}_{1}^{(J K)} \mathcal{C}_{1}^{(K I)}=0 \quad(I \neq J \neq K) \\
& \text { ii) } \omega_{[a i}{ }^{D} \omega_{k] D}{ }^{c}=0 \rightarrow \quad-d_{0}^{(I)} a_{2}^{(J)}+\mathcal{C}_{1}^{(I I)} b_{1}^{(K)}+\mathcal{C}_{1}^{(K I)} b_{1}^{(I)}=0 \quad(I \neq J \neq K) \\
& \text { iii) } \omega_{[i b}^{D} \omega_{c] D}{ }^{7}=0 \rightarrow \\
& \text { iv) } \omega_{[i j}^{D} \omega_{k] D}{ }^{7}=0 \rightarrow \\
& \text { v) } \omega_{[7 a}^{D} \omega_{b] D}^{k}=0 \rightarrow \\
& \sum_{L} a_{2}^{(L)} \mathcal{C}_{1}^{(L I)}=0 \\
& \sum_{K}^{L} b_{1}^{(K)} a_{2}^{(K)}=0 \\
& \sum_{L}^{K} \mathcal{C}_{1}^{(I L)} c_{3}^{(L)}=0
\end{aligned}
$$

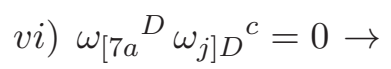

$$
\begin{aligned}
& b_{1}^{(I)} c_{3}^{(J)}+\mathcal{C}_{1}^{(I I)} d_{0}^{(K)}+\mathcal{C}_{1}^{(I K)} d_{0}^{(I)}=0 \quad(I \neq J \neq K) \\
& \text { vii) } \omega_{[7 i}^{D} \omega_{j] D}^{k}=0 \rightarrow \\
& b_{1}^{(I)} c_{3}^{(I)}+\mathcal{C}_{1}^{(I J)} d_{0}^{(K)}+\mathcal{C}_{1}^{(I K)} d_{0}^{(J)}=0 \quad(I \neq J \neq K)
\end{aligned}
$$

whereas the second quadratic constraint in (3.1) is automatically satisfied due to the orbifold symmetries. This can be straightforwardly verified using the M-theory fluxes in table 1.

The application of the isotropic limits (2.40) and (2.41) to the flux parameters reduces the set of quadratic constraints in (3.2) to only 7 conditions. These are given by

$$
\begin{aligned}
& \text { i) } \omega_{[a i}{ }^{D} \omega_{c] D}{ }^{k}=0 \rightarrow-a_{2} c_{3}^{\prime}+c_{1}\left(c_{1}-\tilde{c}_{1}\right)=0 \\
& \text { ii) } \omega_{[a i}^{D} \omega_{k] D}{ }^{c}=0 \rightarrow-d_{0} a_{2}+\left(c_{1}-\tilde{c}_{1}\right) b_{1}=0 \\
& \text { iii) } \omega_{[i b}^{D} \omega_{c] D}{ }^{7}=0 \rightarrow \quad a_{2}\left(2 c_{1}-\tilde{c}_{1}\right)=0 \\
& \text { iv) } \omega_{[i j}{ }^{D} \omega_{k] D}{ }^{7}=0 \rightarrow \quad 3 b_{1} a_{2}=0 \\
& \text { v) } \omega_{[7 a}^{D} \omega_{b] D}{ }^{k}=0 \rightarrow \quad\left(2 c_{1}-\tilde{c}_{1}\right) c_{3}^{\prime}=0 \\
& \text { vi) } \omega_{[7 a}{ }^{D} \omega_{j] D}{ }^{c}=0 \rightarrow b_{1} c_{3}^{\prime}+\left(c_{1}-\tilde{c}_{1}\right) d_{0}=0 \\
& \text { vii) } \omega_{[7 i}{ }^{D} \omega_{j] D}{ }^{k}=0 \rightarrow \quad b_{1} c_{3}^{\prime}+2 c_{1} d_{0}=0 \text {. }
\end{aligned}
$$

We will investigate the connection between the set of quadratic constraints in (3.3) and those required if demanding $\mathcal{N}=8$ or $\mathcal{N}=4$ supersymmetry in the effective action. We will discuss it in the framework of the embedding tensor [3].

\subsection{Extended supersymmetry and gaugings}

The M-theory superpotential in (2.18) is an holomorphic function of the moduli fields and therefore completely unrestricted from the point of view of $\mathcal{N}=1$ supergravity. However, a higher-dimensional origin as an ordinary Scherk-Schwarz reduction of M-theory demands

\footnotetext{
${ }^{11}$ In the expressions (3.2) the $I \neq J \neq K$ assignments are understood in two different manners. For conditions coming in a triplet (multiplicity 3 ) they are understood in a cyclic manner as before, namely $(I, J, K)=(1,2,3),(2,3,1),(3,1,2)$. For conditions coming in a sextuplet (multiplicity 6$)$ they are understood as permutations, namely $(I, J, K)=(1,2,3),(2,1,3),(2,3,1),(3,2,1),(3,1,2),(1,3,2)$.
} 
the additional constraints in (3.2) to be satisfied. We will show now that these conditions are in one-to-one correspondence with the quadratic constraints on the embedding tensor of $\mathcal{N}=8$ supergravity.

Let us start with an intermediate theory between minimal $\mathcal{N}=1$ and maximal $\mathcal{N}=8$ supergravity: the half-maximal $\mathcal{N}=4$ supergravity theory coupled to six vector multiplets. This theory has a global symmetry group $G=\mathrm{SL}(2) \times \mathrm{SO}(6,6)$ reflecting the putative $\mathrm{S}$ and $\mathrm{T}$ dualities of string theory upon toroidal reduction. From a purely supergravity point of view, the flux parameters entering the M-theory superpotential (2.18) determine what is called a gauging or deformation of the $\mathcal{N}=4$ free theory. After applying a gauging, a non-abelian gauge symmetry $G_{0} \subset G$ emerges in the effective action. The gauge algebra is specified by the commutation relations

$$
\left[T_{\alpha M} T_{\beta N}\right]=f_{\alpha M N}{ }^{P} T_{\beta P},
$$

where $T_{\alpha M}$ denotes the generators associated to the non-abelian vector fields - indices $\alpha=+,-$ and $M=1, \ldots, 12$ are respectively fundamental $\mathrm{SL}(2)$ and $\mathrm{SO}(6,6)$ indices and $f_{\alpha M N}{ }^{P}$ (structure constants) is the so-called embedding tensor (ET).

The M-theory fluxes in (2.18) can be mapped to different components of the embedding tensor. To be more precise, this connection was established $[8,9]$ in a type IIA (and also IIB) incarnation of the four-dimensional STU-model defined by (2.18). Using light-cone coordinates for the $\mathrm{SO}(6,6)$ fundamental index $M$ amounts to choosing

$$
\eta=\left(\begin{array}{cc}
0 & \mathbb{I}_{6} \\
\mathbb{I}_{6} & 0
\end{array}\right)
$$

as the invariant metric to raise and lower $\mathrm{SO}(6,6)$ indices. If we further split the index $M$ as $M=\left(a,{ }_{i},{ }^{a},{ }^{i}\right)$, then the fluxes/ET dictionary is presented in table 3 . Notice the presence of electric $(\alpha=+)$ as well as magnetic $(\alpha=-)$ components within the embedding tensor $f_{\alpha M N P}=f_{\alpha M N}{ }^{Q} \eta_{Q P}$. Both are simultaneously required in order to avoid a runaway behaviour for the dilaton modulus [34].

The consistency of a gauging in $\mathcal{N}=4$ supergravity [3] imposes a set of quadratic constraints on the embedding tensor $f_{\alpha M N P}$. These are given by

$$
f_{\alpha R[M N} f_{\beta P Q]}^{R}=0 \quad \text { and } \quad \epsilon^{\alpha \beta} f_{\alpha M N R} f_{\beta P Q}^{R}=0
$$

where $\epsilon^{\alpha \beta}=\epsilon_{\alpha \beta}$ with $\epsilon^{+-}=-\epsilon^{-+}=1$ is used to raise and lower the $\operatorname{SL}(2)$ index $\alpha$. In order to make contact with the Scherk-Schwarz conditions in (3.1) for M-theory reductions, we have to set the Romans mass to zero, i.e. $a_{3}=0$, among the fluxes in table 3 as it corresponds to a non-geometric flux in M-theory. The explicit computation of the constraints in (3.6) produces the following conditions

$$
\begin{aligned}
f_{\alpha R[M N} f_{\beta P Q]}{ }^{R} & =0 \rightarrow \text { Conditions } i), \text { iii) and } v) \text { in }(3.2), \\
\epsilon^{\alpha \beta} f_{\alpha M N R} f_{\beta P Q}{ }^{R} & =0 \rightarrow \text { Conditions } i i) \text { and } v i) \text { in }(3.2) .
\end{aligned}
$$

As a result, the quadratic constraints of $\mathcal{N}=4$ supergravity (3.6) fail to reproduce the two additional conditions $i v$ ) and vii) in (3.2). Therefore, demanding $\mathcal{N}=4$ in the effective 


\begin{tabular}{|c|c|c|c|}
\hline M-theory origin & Type IIA origin & Fluxes & Embedding tensor \\
\hline$\omega_{b c}^{a}$ & $\omega_{b c}^{a}$ & $\tilde{c}_{1}^{(I)}$ & $f_{+}^{b c} a$ \\
\hline$\omega_{a j}^{k}$ & $\omega_{a j}^{k}$ & $\hat{c}_{1}^{(I)}$ & $f_{+}^{a j}$ \\
\hline$\omega_{k a^{j}}$ & $\omega_{k a^{j}}$ & $\check{c}_{1}^{(I)}$ & $f_{+}^{k a}{ }_{j}$ \\
\hline$\omega_{j k}{ }^{a}$ & $\omega_{j k}{ }^{a}$ & $b_{1}^{(I)}$ & $f_{-} i b c$ \\
\hline$-\omega_{a i}{ }^{7}$ & $F_{a i}$ & $a_{2}^{(I)}$ & $-f_{+}^{a j k}$ \\
\hline$-\omega_{7 i}^{a}$ & non-geometric & $d_{0}^{(I)}$ & $f_{-}{ }^{b c} i$ \\
\hline$-\omega_{a 7^{i}}$ & non-geometric & $c_{3}^{\prime(I)}$ & $f_{+j k}^{a}$ \\
\hline$-\frac{1}{2} G_{a i b j}$ & $-F_{a i b j}$ & $a_{1}^{(I)}$ & $f_{+}^{a b k}$ \\
\hline$\frac{1}{2} G_{i j k 7}$ & $H_{i j k}$ & $b_{0}$ & $-f_{-}^{a b c}$ \\
\hline$\frac{1}{2} G_{i b c 7}$ & $H_{i b c}$ & $c_{0}^{(I)}$ & $f_{+}{ }^{b c} i$ \\
\hline$\frac{1}{4} G_{a i b j c k 7}$ & $F_{\text {aibjck }}$ & $a_{0}$ & $-f_{+}^{a b c}$ \\
\hline non-geometric & $-F_{(0)} \quad$ (Romans mass) & $a_{3}$ & $f_{+}^{i j k}$ \\
\hline
\end{tabular}

Table 3. M-theory/type IIA fluxes and embedding tensor.

theory is less restrictive than demanding a higher-dimensional interpretation as an ordinary Scherk-Schwarz reduction of M-theory.

In ref. [35] it was shown that the $\mathcal{N}=4$ constraints (3.6) must be supplemented with two additional ones

$$
\left.\epsilon^{\alpha \beta} f_{\alpha[M N P} f_{\beta Q R S]}\right|_{\mathrm{SD}}=0 \quad \text { and } \quad f_{\alpha M N P} f_{\beta}{ }^{M N P}=0
$$

in order to have an $\mathcal{N}=4 \rightarrow \mathcal{N}=8$ supersymmetry enhancement in the effective action. The label SD in the first constraint in (3.8) restricts it to the self-dual part of the $\mathrm{SO}(6,6)$ six-form $\epsilon^{\alpha \beta} f_{\alpha[M N P} f_{\beta Q R S]}$. Once more, an explicit computation of these two constraints produces

$$
\begin{aligned}
\left.\epsilon^{\alpha \beta} f_{\alpha[M N P} f_{\beta Q R S]}\right|_{\mathrm{SD}} & =0 \rightarrow \text { Conditions } i v) \text { and vii) in }(3.2), \\
f_{\alpha M N P} f_{\beta} M N P & =0 \rightarrow \text { No additional conditions }
\end{aligned}
$$

hence completing the set of conditions in (3.2). In other words, there is a one-to-one correspondence between the $\mathcal{N}=8$ quadratic constraints and the conditions required by an ordinary Scherk-Schwarz reduction of M-theory.

\subsection{KK6 monopoles and $\mathcal{N}=8 \rightarrow \mathcal{N}=4$ breaking}

In the previous section we have seen that requiring an $\mathcal{N}=4$ description of the effective supergravity allows for a relaxation of the conditions $i v$ ) and vii) in (3.2). However these 
still have to be imposed in any ordinary Scherk-Schwarz reduction of M-theory establishing the link to $\mathcal{N}=8$ supergravity.

On the other hand, a violation of some of the $\omega \omega=0$ conditions in (3.1) has been connected to the presence of KK6 monopoles in the compactification scheme, thus going beyond twisted tori [6]. From the effective field theory point of view, we will refer to the would-be companion sources carrying negative charge as KKO6-planes following a similar terminology to that of ref. [6]. Schematically,

$$
\left.\omega_{[\bullet \bullet}^{D} \omega_{\bullet}\right] D=0 \Rightarrow \text { Non-vanishing KK6 (KKO6) charge, }
$$

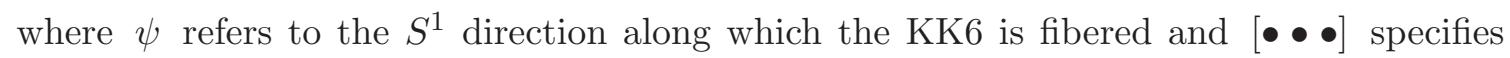
the 3 -form dual to the 7 -cycle filled by the KK6 and the $S^{1}$ fiber. The KK6 monopoles will induce a positive contribution to the scalar potential whereas the one coming from the KKO6-planes will be negative [6].

In the case of $X_{7}=\mathbb{T}^{7} /\left(\mathbb{Z}_{2} \times \mathbb{Z}_{2} \times \mathbb{Z}_{2}\right)$, there are 28 different KK6 monopoles compatible with the orbifold symmetries. These KK6's can be grouped as $6+6+3+1+3+6+$ $3=28$ and source the r.h.s. of the set of conditions in (3.2). KK6 monopoles in Mtheory sourcing the $6+6$ conditions $i$ ) and $i i$ ) give rise to KK5's (fibered over $\eta^{i}$ ) and $\widetilde{\mathrm{KK} 5}$ 's (fibered over $\eta^{a}$ ) monopoles in type IIA upon dimensional reduction. Those fibering $\eta^{7}$ source the $3+1$ conditions $i i i$ ) and $i v$ ) and give rise to D6 $\perp$ 's (threading 3 -cycles $\eta^{a j k}$ ) and D6, 's (threading the 3-cycle $\eta^{a b c}$ ) upon reduction to type IIA along the $\eta^{7}$ direction. There are also $3+6+3$ KK6 monopoles sourcing the conditions $v$ ), vi) and vii) which do not have an interpretation as type IIA sources. We denote them KK6 $\perp$ 's and $\widetilde{\mathrm{KK}}{ }_{\perp}$ 's (threading 3-cycles $\eta^{a j k}$ and respectively fibered over $\eta^{i}$ and $\eta^{a}$ ) as well as KK6 $\|_{\|}$'s (threading the 3 -cycle $\eta^{a b c}$ and fibered over $\eta^{i}$ ). By looking at the conditions in (3.2), a non-vanishing net charge of $\mathrm{KK} 6_{\perp}$ 's, $\widetilde{\mathrm{KK} 6} 6_{\perp}$ 's and $\mathrm{KK} 6{ }_{\|}$'s requires a non-trivial background for the fluxes $\left(c_{3}^{\prime(I)}, d_{0}^{I}\right)$. These are the M-theory fluxes without a type IIA counterpart in table 2, thus corresponding to non-geometric type IIA flux backgrounds. For the set of conditions in (3.2), the corresponding types of KK6 monopoles are summarised in table 4.

Our last concern is that of supersymmetry breaking in the presence of KK6 monopoles. From the general discussion of quadratic constraints in $\mathcal{N}=4,8$ supergravity of the previous section, the effective theory preserves $\mathcal{N}=8$ supersymmetry only if no KK6 net charge is induced by the M-theory flux backgrounds. In this case the full set of conditions in (3.6) and (3.8) are satisfied implying an ordinary Scherk-Schwarz reduction of M-theory with no violation of the constraints (3.1). If the M-theory background fluxes induce a nonvanishing charge for KK6 (KKO6) monopoles corresponding to D6 $6_{\|}\left(\mathrm{O} 6_{\|}\right), \mathrm{KK} 6_{\|}\left(\mathrm{KKO} 6_{\|}\right)$ or both, then $\mathcal{N}=4$ supersymmetry is still preserved but one goes beyond Scherk-Schwarz reductions of M-theory due to the violation of (3.1). We will exhaustively explore these two types of effective theories in the next section.

\subsection{Universal IIA moduli, KK6 monopoles and scalar potential}

A way of understanding the effect of including M-theory sources in the background is to analyse the moduli powers appearing in the scalar potential. In order to make contact with 


\begin{tabular}{|c|c|c|c|c|c|c|c|c|c|c|c|c|c|}
\hline Type & $x^{0}$ & $x^{1}$ & $x^{2}$ & $x^{3}$ & $\eta^{a}$ & $\eta^{i}$ & $\eta^{b}$ & $\eta^{j}$ & $\eta^{c}$ & $\eta^{k}$ & $\eta^{7}$ & $\mathrm{KK} 6 \rightarrow$ type IIA & $\mathcal{N}=4 ?$ \\
\hline$i)$ & $\times$ & $\times$ & $\times$ & $\times$ & $\times$ & $\times$ & & $\psi$ & & & $\times$ & $\mathrm{KK} 5(\mathrm{KKO} 5)$ & no \\
\hline$i i)$ & $\times$ & $\times$ & $\times$ & $\times$ & $\times$ & $\times$ & $\psi$ & & & & $\times$ & $\widetilde{\mathrm{KK} 5}(\widetilde{\mathrm{KKO} 5})$ & no \\
\hline$i i i)$ & $\times$ & $\times$ & $\times$ & $\times$ & $\times$ & & & $\times$ & & $\times$ & $\psi$ & $\mathrm{D} 6_{\perp}\left(\mathrm{O} 6_{\perp}\right)$ & no \\
\hline$i v)$ & $\times$ & $\times$ & $\times$ & $\times$ & $\times$ & & $\times$ & & $\times$ & & $\psi$ & $\mathrm{D} 6_{\|}\left(\mathrm{O} 6_{\|}\right)$ & yes \\
\hline$v)$ & $\times$ & $\times$ & $\times$ & $\times$ & $\times$ & $\psi$ & & $\times$ & & $\times$ & & $\mathrm{KK}_{\perp}\left(\mathrm{KKO}_{\perp}\right)$ & no \\
\hline$v i)$ & $\times$ & $\times$ & $\times$ & $\times$ & $\times$ & & $\psi$ & $\times$ & & $\times$ & & $\widetilde{\mathrm{KK}} 6_{\perp}\left(\widetilde{\mathrm{KKO}} 6_{\perp}\right)$ & no \\
\hline$v i i)$ & $\times$ & $\times$ & $\times$ & $\times$ & $\times$ & & $\times$ & & $\times$ & $\psi$ & & $\mathrm{KK}_{\|}\left(\mathrm{KKO}_{\|}\right)$ & yes \\
\hline
\end{tabular}

Table 4. Set of KK6 (KKO6) monopoles compatible with the $X_{7}=\mathbb{T}^{7} /\left(\mathbb{Z}_{2} \times \mathbb{Z}_{2} \times \mathbb{Z}_{2}\right)$ orbifold. They respectively source the r.h.s. of the set of conditions in (3.2). Only D6 $\|\left(\mathrm{O} 6_{\|}\right)$and $\mathrm{KK} 6_{\|}$ $\left(\mathrm{KKO}_{\|}\right)$sources can be consistently introduced in a background preserving $\mathcal{N}=4$ supersymmetry in four dimensions.

previous results in the literature [36-40] we will reinterpret the M-theory potential from a type IIA point of view. To this end, let us introduce the three universal IIA moduli fields $(\tau, \rho, \sigma)$ entering the $10 \mathrm{~d}$ metric

$$
d s_{10}^{2}=\tau^{-2} d s_{4}^{2}+\rho\left(\sigma^{-3} M_{a b} d y^{a} d y^{b}+\sigma^{3} M_{i j} d y^{i} d y^{j}\right),
$$

which are related to the STU fields as

$$
\tau=\operatorname{Im}(S)^{1 / 4} \operatorname{Im}(T)^{3 / 4}, \quad \rho=\operatorname{Im}(U), \quad \sigma=\operatorname{Im}(S)^{-1 / 6} \operatorname{Im}(T)^{1 / 6} .
$$

We follow the conventions in appendix B of ref. [41] regarding dimensional reduction of 10d type IIA supergravity.

Setting the axions to zero, namely $\operatorname{Re}(S)=\operatorname{Re}(T)=\operatorname{Re}(U)=0$, the computation of the scalar potential from the M-theory superpotential (2.42) reveals the following $\tau$ dependence structure

$$
V_{\mathrm{M} \text {-theory }}(\tau, \rho, \sigma)=\frac{1}{32} \sum_{n=0}^{4} V_{n}(\tau, \rho, \sigma)=\frac{1}{32} \sum_{n=0}^{4} A_{n}(\rho, \sigma) \tau^{-n} .
$$

The functions $A_{n}(\rho, \sigma)$ that determine the different terms $V_{n}=A_{n}(\rho, \sigma) \tau^{-n}$ in the potential take the following form:

$$
\begin{aligned}
A_{0}(\rho, \sigma)= & 3 \rho^{-3}\left(c_{3}^{\prime} \sigma^{3}-d_{0} \sigma^{-3}\right)^{2} \\
A_{1}(\rho, \sigma)= & 6 \rho^{-2}\left[\left(2 c_{1}-\tilde{c}_{1}\right) c_{3}^{\prime} \sigma^{9 / 2}+2\left(b_{1} c_{3}^{\prime}+\left(c_{1}-\tilde{c}_{1}\right) d_{0}\right) \sigma^{-3 / 2}+\left(b_{1} c_{3}^{\prime}+2 c_{1} d_{0}\right) \sigma^{-3 / 2}\right] \\
A_{2}(\rho, \sigma)= & \rho^{-3}\left(b_{0}^{2} \sigma^{-9}+3 c_{0}^{2} \sigma^{3}\right)+3 \rho^{-1}\left[b_{1}^{2} \sigma^{-9}-4 b_{1}\left(2 c_{1}-\tilde{c}_{1}\right) \sigma^{-3}-\left(2 c_{1}-\tilde{c}_{1}\right)^{2} \sigma^{3}\right] \\
& +18 \rho^{-1} a_{2}\left(c_{3}^{\prime} \sigma^{3}+d_{0} \sigma^{-3}\right) \\
A_{3}(\rho, \sigma)= & -6\left(2 c_{1}-\tilde{c}_{1}\right) a_{2} \sigma^{3 / 2}-6 a_{2} b_{1} \sigma^{-9 / 2} \\
A_{4}(\rho, \sigma)= & a_{0}^{2} \rho^{-3}+3 a_{1}^{2} \rho^{-1}+3 a_{2}^{2} \rho .
\end{aligned}
$$


Let us discuss the $V_{n}$ terms in the M-theory scalar potential (3.13) when adopting a type IIA point of view using the M-theory/type IIA dictionary in table 3. Recall that only the flux parameters $\left(c_{3}^{\prime}, d_{0}\right)$ are genuine M-theory metric fluxes without type IIA counterparts. These fluxes are responsible for the two terms $V_{0} \propto \tau^{0} \rho^{-3}$ and $V_{1} \propto \tau^{-1} \rho^{-2}$ which have no analogous in a regular IIA orientifold model $[36,39,40]$ thus corresponding to nongeometric contributions in a IIA incarnation of the potential (3.13). The three pieces inside $V_{1}$ account for the net charge of $\mathrm{KK}_{\perp}\left(\mathrm{KKO} 6_{\perp}\right), \widetilde{\mathrm{KK}} 6_{\perp}\left(\widetilde{\mathrm{KKO}} 6_{\perp}\right)$ and $\mathrm{KK} 6_{\|}\left(\mathrm{KKO} 6_{\|}\right)$ monopoles, respectively. The term $V_{2}$ plays a central role in stabilising moduli and contains two types of contributions proportional to $\tau^{-2} \rho^{-3}$ and $\tau^{-2} \rho^{-1}$ respectively: the former is sourced by NS-NS fluxes $H_{(3)}$ in the IIA picture (first line in $A_{2}$ ) whereas the latter is induced by metric IIA fluxes $\omega_{(\mathrm{IIA})}$ (second line in $A_{2}$ ) as well as the two fluxes $\left(c_{3}^{\prime}, d_{0}\right)$ which are non-geometric in the IIA description of the STU-model (third line in $A_{2}$ ). The two pieces in $V_{3} \propto \tau^{-3} \rho^{0}$ respectively account for the net charge of $\mathrm{D} 6_{\perp}$ and $\mathrm{D} 6_{\|}$sources and the corresponding orientifold planes. Finally the $V_{4}$ term contains the type IIA R$\mathrm{R}$ contributions to the scalar potential. Notice the absence of the Romans contribution $V_{\text {Romans }} \propto \tau^{-4} \rho^{3}$ that would be induced by the flux parameter $a_{3}$ which is not present in the M-theory setup and played a central role in the construction of ref. [38] producing de Sitter $\left(\mathrm{dS}_{4}\right)$ solutions.

In the previous section we saw that including a net charge for those KK6 sources in M-theory which correspond to KK5 and $\widetilde{\mathrm{KK} 5}$ monopoles in the IIA picture - types $i$ ) and $i$ i) in table 4 - was not compatible with preserving $\mathcal{N}=4$ supersymmetry in the effective action. The reason was that the associated conditions $i$ ) and ii) in (3.3) still hold after relaxing $\mathcal{N}=8 \rightarrow \mathcal{N}=4$. The effect of adding such monopoles has been investigated in refs $[37,38]$ and found to induce an extra piece $V_{\mathrm{KK} 5} \propto \tau^{-2} \rho^{-1}$ in the potential supplementing the one already induced by the IIA metric flux $V_{\omega_{\text {IIA }}} \propto \tau^{-2} \rho^{-1}$ with the same moduli powers. More importantly, this extra piece $V_{\mathrm{KK} 5}$ turned out to help in finding de Sitter solutions [37,38]. Even though we cannot include such KK5 and $\widetilde{\mathrm{KK} 5}$ monopoles when demanding $\mathcal{N}=4$ supersymmetry, the M-theory fluxes $\left(c_{3}^{\prime}, d_{0}\right)$ will potentially induce the desired $\tau^{-2} \rho^{-1}$ extra piece within $V_{2}$ (third line in $\left.A_{2}\right) .{ }^{12}$ Despite this promising fact, only Anti-de Sitter $\left(\mathrm{AdS}_{4}\right)$ solutions will happen to exist in these $\mathcal{N}=4$ STU-models.

\section{Taxonomy of M-theory flux vacua}

In this section we will exhaustively classify the entire set of critical points of the scalar potential obtained from the M-theory superpotential (2.42) when demanding $\mathcal{N}=4$ supersymmetry in the effective action. In addition to the superpotential analysis, we have

\footnotetext{
${ }^{12}$ This $\tau^{-2} \rho^{-1}$ extra piece within $V_{2}$ can be obtained from the M-theory superpotential (2.42) but not from the type IIA superpotential (2.43) due to the lack of the two relevant fluxes $\left(c_{3}^{\prime}, d_{0}\right)$. This result also holds after turning on the three STU axions as they do not modify the second and third lines in $A_{2}(\rho, \sigma)$. Only $A_{4}(\rho, \sigma)$ and the first line in $A_{2}(\rho, \sigma)$ corresponding to R-R and NS-NS gauge fluxes in the IIA picture are modified by the STU axions.
} 
also verified all the results by explicit computations using the $\mathcal{N}=4$ scalar potential directly built from the embedding tensor [3].

\subsection{Exploiting dualities in the effective theory}

The $\mathcal{N}=1$ supergravity model we derived in section 2.1 can be formally viewed as a discrete $\mathbb{Z}_{2} \times \mathbb{Z}_{2}$ truncation of the $\mathcal{M}_{\text {scalar }}^{\mathcal{N}=4}=[\mathrm{SL}(2) / \mathrm{SO}(2)] \times[\mathrm{SO}(6,6) / \mathrm{SO}(6) \times \mathrm{SO}(6)]$ coset space spanned by the $2+36$ scalar fields of the $\mathcal{N}=4$ theory. The seven complex moduli $T_{A}=\left(S, T_{I}, U_{I}\right)$ correspond to the seven dilatons (Cartan generators) as well as seven axions (positive roots), and span the Kähler manifold $\mathcal{M}_{\text {scalar }}^{\text {(non-iso }}=[\mathrm{SU}(1,1) / \mathrm{U}(1)]^{7}$. As we already discussed, demanding isotropy imposes an additional "plane exchange" $\mathrm{SO}(3)$ cyclic symmetry among the three two-tori in $\mathbb{T}^{6}=\mathbb{T}^{2} \times \mathbb{T}^{2} \times \mathbb{T}^{2}$ (inside $X_{7}$ ). This additional symmetry can be interpreted as an enhancement of the truncation from a $\mathbb{Z}_{2} \times \mathbb{Z}_{2}$ truncation to an $\mathrm{SO}(3)$ truncation. After taking into account the isotropic identifications in (2.38), the three complex moduli in the STU-model serve as coordinates in the Kähler submanifold $\mathcal{M}_{\text {scalar }}^{\text {(iso) }}=[\mathrm{SU}(1,1) / \mathrm{U}(1)]^{3} \subset[\mathrm{SU}(1,1) / \mathrm{U}(1)]^{7}$.

The $\mathcal{M}_{\text {scalar }}^{\text {(iso) }}$ coset space is a symmetric space and therefore any point can be connected to any other via a non-compact $\mathrm{SU}(1,1)^{3}$ transformation. The action of this transformation on the STU-model is that of rescaling and shifting the moduli fields as

$$
S \rightarrow \lambda_{S} S+\Delta_{S}, \quad T \rightarrow \lambda_{T} T+\Delta_{T}, \quad U \rightarrow \lambda_{U} U+\Delta_{U}
$$

with $\lambda_{S, T, U}$ and $\Delta_{S, T, U}$ being real parameters. By using the tranformations (4.1), any moduli configuration corresponding to a critical point of the scalar potential can be brought to the origin of the moduli space defined as

$$
S_{0}=T_{0}=U_{0}=i .
$$

After bringing the moduli configuration to the origin (4.2), the associated flux parameters entering the superpotential (2.42) will change in order to leave the scalar potential invariant. Notice that the M-theory superpotential in (2.42) is not only quadratic on the moduli but it also contains the linear dependences as well as the constant term. This ensures that the new flux background obtained after bringing the moduli configuration to the origin will not lie outside the family of STU-models we are considering here. In other words, the M-theory backgrounds form a closed set under the action of the duality tranformations (4.1).

The above argument allows us to look for moduli stabilisation at any point in moduli space and, for the sake of simplicity, we will choose such a point to be the origin (4.2). This does not imply any loss of generality as long as one keeps the complete set of flux parameters in the superpotential (2.42). Focusing on the M-theory backgrounds preserving at least $\mathcal{N}=4$ supersymmetry in the effective action, the structure of critical points can therefore be obtained by solving the algebraic system

$$
\left\langle\left(\text { relaxed) set of conditions in }(3.3),\left.\frac{\partial V}{\partial \phi}\right|_{S_{0}=T_{0}=U_{0}=i}\right\rangle=0,\right.
$$

which consists of quadratic conditions on the flux parameters. As discussed in the previous section, the conditions $i v$ ) and vii) in (3.3) can be consistently relaxed if one goes beyond 
twisted tori reductions but still requires the effective action to have $\mathcal{N}=4$ supersymmetry. The above set of equations in (4.3) can be completely solved - with or without relaxing $i v$ ) and vii) - by using algebraic geometry tools included in the computational package SINGULAR [42]. In particular, we have used the GTZ built-in algorithm for primary decomposition of ideals. The outcome is that (4.3) contains several prime factors, each of which corresponds to a physically different content of KK6 monopoles in the M-theory background. We will discuss them in detail later on.

Some other advantages of bringing the moduli configurations to the origin are: $i$ ) closed expressions for the particle mass spectra at a critical point of the scalar potential have been worked out [43] ii) the fermion mass terms get a much simpler form. The fermion masses can be viewed as "dressing up" the embedding tensor with the moduli dependence [3]. When evaluated at the origin, the $\mathcal{N}=4$ gravitini mass matrix acquires the very simple form

$$
\mathcal{A}=-\frac{3}{8 \sqrt{2}}\left(\begin{array}{cc}
\mathcal{A}_{1} & 0 \\
0 & \mathcal{A}_{2} \mathbb{I}_{3 \times 3}
\end{array}\right),
$$

with the two independent entries given by

$$
\begin{aligned}
& \mathcal{A}_{1}=\left(a_{0}-3 a_{2}-3 b_{1}-3\left(2 c_{1}-\tilde{c}_{1}\right)+3 c_{3}^{\prime}+3 d_{0}\right)+i\left(3 c_{0}-3 a_{1}-b_{0}\right), \\
& \mathcal{A}_{2}=\left(a_{0}+a_{2}+b_{1}+2 c_{1}+3 \tilde{c}_{1}-c_{3}^{\prime}-d_{0}\right)+i\left(a_{1}-b_{0}-c_{0}\right) .
\end{aligned}
$$

Notice that $\mathcal{A}_{1}=W_{\mathrm{M} \text {-Theory }}^{\text {(iso) }}$ at the origin of the moduli space as it has to in order to identify the $\mathcal{N}=1$ gravitino mass with $\left|W_{\text {M-Theory }}^{(\text {iso) }}\right|$ The gravitino mass matrix in (4.4) can be used to determine the amount of supersymmetry preserved at a critical point of the scalar potential. Provided an $\mathrm{AdS}_{4}$ vacuum solution, it will preserve $\mathcal{N}=1$ supersymmetry if $\left|\mathcal{A}_{1}\right|^{2}=-3 V_{0}$ and $\left|\mathcal{A}_{1}\right|^{2} \neq\left|\mathcal{A}_{2}\right|^{2}$. Similarly, it will preserve $\mathcal{N}=3$ supersymmetry if $\left|\mathcal{A}_{2}\right|^{2}=-3 V_{0}$ and $\left|\mathcal{A}_{1}\right|^{2} \neq\left|\mathcal{A}_{2}\right|^{2}$. Finally it will preserve $\mathcal{N}=4$ supersymmetry if $\left|\mathcal{A}_{1}\right|^{2}=\left|\mathcal{A}_{2}\right|^{2}=-3 V_{0}$ and will be non-supersymmetric otherwise.

\subsection{Backgrounds without KK6 (KKO6)}

Let us start by studying the case of not having KK6 monopoles of any type. Therefore, the full set of conditions in (3.3) have to be imposed and maximal $\mathcal{N}=8$ supersymmetry is preserved in the effective action. The M-theory flux background solving (4.3) in this case is given by

$$
a_{0}=a_{1}=a_{2}=b_{0}=b_{1}=c_{0}=c_{1}=\tilde{c}_{1}=0 \quad \text { and } \quad c_{3}^{\prime}=d_{0}=\lambda,
$$

so that the M-theory superpotential in (2.42) takes the simple form

$$
W_{\text {M-Theory }}^{(\text {iso) }}=-3 \lambda T(S+T) .
$$

Within the class of $\mathcal{N}=1$ STU-models, this superpotential specifies a no-scale supergravity so the vacuum corresponds to a non-supersymmetric and Minkowski $\left(V_{0}=0\right)$ critical point with flat directions. The analysis of the torsion classes shows non-vanishing $\widetilde{W}_{1}$ and $\widetilde{W}_{27}$, thus specifying a general co-calibrated $G_{2}$-structure $(2.19)$. 
The associated M-theory background only contains non-geometric fluxes $\left(c_{3}^{\prime}, d_{0}\right)$ in a type IIA incarnation. This is compatible with the results found in refs $[4,8,31,44]$ regarding type IIA moduli stabilisation based on the superpotential (2.43). In ref. [31] an exhaustive classification of vacua compatible with the full set (3.3) of $\mathcal{N}=8$ constraints (after setting $c_{3}^{\prime}=d_{0}=0$ ) showed the necessity of a non-vanishing Romans mass $\left(a_{3} \neq 0\right)$ in order to achieve full moduli stabilisation. The Romans flux parameter in type IIA does not descend directly from M-theory (see table 3), so the IIA solutions in ref. [31] will not appear in an M-theory context. Finally the M-theory $\omega$-twist corresponds to $G_{\omega}=\operatorname{Solv}_{6} \rtimes \mathrm{U}(1)$ in agreement with the analysis of twist groups performed in ref. [12].

\subsection{Backgrounds with KK6 (KKO6)}

We have rederived the result that there is no moduli stabilisation (without flat directions) in the absence of KK6 (KKO6) monopoles [12]. Next step is then to remove the conditions

$$
\begin{aligned}
& \text { iv) } \quad \omega_{[i j}^{D} \omega_{k] D}{ }^{7}=0 \quad \rightarrow \quad 3 b_{1} a_{2}=0 \text {, } \\
& \text { vii) } \quad \omega_{[7 i}^{D} \omega_{j] D}{ }^{k}=0 \rightarrow b_{1} c_{3}^{\prime}+2 c_{1} d_{0}=0 \text {, }
\end{aligned}
$$

from the system (3.3) in order to preserve only $\mathcal{N}=4$ and investigate the physical implications. Running the primary decomposition algorithm for the relaxed algebraic system in (4.3) one finds three prime factors they all of dimension one. We will discuss each of them separately and show how full moduli stabilisation can take place in M-theory backgrounds containing KK6 (KKO6) monopoles.

\subsubsection{Including only KK6 (KKO6) $\rightarrow \mathrm{D} 6_{\|}\left(\mathrm{O} 6_{\|}\right)$sources}

The first prime factor in the decomposition of (4.3) is compatible with a relaxation of the condition

$$
\text { iv) } \quad \omega_{[i j}^{D} \omega_{k] D}{ }^{7} \neq 0 \rightarrow 3 b_{1} a_{2} \neq 0,
$$

whereas the rest of conditions in (3.3) are still satisfied. This case is then interpreted as an M-theory background which only includes those KK6 (KKO6) monopoles that can be interpreted as $\mathrm{D} 6_{\|}\left(\mathrm{O} 6_{\|}\right)$type IIA sources upon reduction.

By explicitly solving this prime factor we find a one dimensional family of M-theory flux backgrounds of the form

$$
a_{0}=b_{0}=a_{1}=c_{0}=c_{1}=\tilde{c}_{1}=c_{3}^{\prime}=d_{0}=0 \quad \text { and } \quad b_{1}=a_{2}=\lambda .
$$

After substitution into (2.42), the M-theory superpotential reads

$$
W_{\mathrm{M} \text {-Theory }}^{\text {(iso) }}=3 \lambda U(S+U),
$$

corresponding to a no-scale STU-model analogous to that in (4.7) upon exchanging $T \leftrightarrow$ $U$. The associated vacuum - we will refer to it as "vac 0" from now on - is a non-supersymmetric Minkowski vacuum with non-vanishing $\widetilde{W}_{1}$ and $\widetilde{W}_{27}$ torsion classes in (2.19). 
Using the mass formula in ref. [43], the scalar mass spectrum is given by

$$
m^{2}=\frac{9}{8} \lambda^{2}(\times 1), \quad \frac{1}{2} \lambda^{2}(\times 6), \frac{1}{8} \lambda^{2}(\times 9), 0(\times 22),
$$

so it does not contain tachyons but presents thirteen flat directions, i.e., zero-mass modes not associated to Goldstone bosons. The spectrum of vector masses reads

$$
m^{2}=\frac{1}{2} \lambda^{2}(\times 3), \frac{1}{8} \lambda^{2}(\times 6), 0(\times 3),
$$

and contains three massless vectors reflecting the residual $G_{\text {res }}=\mathrm{SO}(3)$ cyclic symmetry of the isotropic STU-model.

In a type IIA interpretation of this M-theory flux vacuum, we have introduced $\mathrm{D} 6_{\|} / \mathrm{O} 6_{\|}$ sources in the background wrapping the 3-cycle $\eta^{a b c}$ in order to cancel a flux-induced tadpole for the R-R gauge potential $C_{(7)}$. The BI for $F_{(2)}$ along the internal space $X_{6}$ reads

$$
d F_{(2)}=\omega F_{(2)}=N_{\mathrm{O}_{\|} \|}-N_{\mathrm{D} 6_{\|}}=3 b_{1} a_{2}=3 \lambda^{2}>0,
$$

thus demanding $\mathrm{O}_{\|}$orientifold planes lifting to KKO6-planes in M-theory (see ref. [6] and references therein for a discussion of the lifting).

\subsubsection{Including only $\mathrm{KK} 6(\mathrm{KKO} 6) \rightarrow \mathrm{KK} 6_{\|}\left(\mathrm{KKO} 6_{\|}\right)$sources}

The second prime factor in the decomposition of the algebraic system (4.3) is compatible with relaxing

$$
\text { vii) } \quad \omega_{[7 i}^{D} \omega_{j] D}{ }^{k} \neq 0 \rightarrow b_{1} c_{3}^{\prime}+2 c_{1} d_{0} \neq 0
$$

but still requires the rest of the conditions in (3.3) to vanish. Therefore, the resulting M-theory backgrounds only include $\mathrm{KK} 6_{\|}\left(\mathrm{KKO} 6_{\|}\right)$monopoles. Backgrounds including a net charge of these objects do not admit a description in terms of ordinary type IIA orientifolds. Instead, they correspond to non-geometric type IIA backgrounds.

Solving this prime factor explicitly reveals a rich structure of M-theory flux vacua they all compatible with

$$
a_{2}=c_{3}^{\prime}=0 \quad \text { and } \quad c_{1}=\tilde{c}_{1}=\lambda,
$$

so that the $U^{2}$ and $T^{2}$ terms in the superpotential (2.42) are absent. Up to some discrete multiplicities there are eight inequivalent vacua we have denoted "vac 1" to "vac 8". The physical implications of these M-theory backgrounds are very diverse and we have carried out a detailed analysis in appendix A. A brief summary of the main results is presented also in table 5 . In all the solutions the net charge of $\mathrm{KK} 6_{\|}\left(\mathrm{KKO} 6_{\|}\right)$sources is

$$
N_{\mathrm{KK} 6_{\|}}-N_{\mathrm{KKO}_{\|}}=b_{1} c_{3}^{\prime}+2 c_{1} d_{0}<0,
$$

hence requiring $\mathrm{KKO} 6_{\|}$-planes to be present in the background. At this point we want to highlight that full moduli stabilisation at supersymmetric as well as at non-supersymmetric vacua is achieved for some of these M-theory backgrounds. 


\begin{tabular}{|c|c|c|c|c|c|c|c|}
\hline ID & STU-model & $\mathrm{D} 6_{\|}\left(\mathrm{O} 6_{\|}\right) / \mathrm{KK} 6_{\|}\left(\mathrm{KKO} 6_{\|}\right)$ & Stable & Flat dir. & SUSY & $\operatorname{dim}\left(G_{\text {res }}\right)$ & $\widetilde{W}_{27}$ \\
\hline vac 0 & no-scale & yes / no & $\checkmark$ & yes & $\mathcal{N}=0$ & 3 & $\neq 0$ \\
\hline $\operatorname{vac} 1$ & Model 1 & no / yes & $\checkmark$ & yes & $\mathcal{N}=3$ & 3 & $\neq 0$ \\
\hline $\operatorname{vac} 2$ & Model 2 & no / yes & $\checkmark$ & yes & $\mathcal{N}=0$ & 3 & $\neq 0$ \\
\hline $\operatorname{vac} 3$ & Model 1 & no / yes & $\checkmark$ & no & $\mathcal{N}=0$ & 3 & 0 \\
\hline $\operatorname{vac} 4$ & Model 2 & no / yes & $\checkmark$ & no & $\mathcal{N}=1$ & 3 & 0 \\
\hline $\operatorname{vac} 5$ & Model 1 & no / yes & $\checkmark$ & no & $\mathcal{N}=0$ & 3 & 0 \\
\hline $\operatorname{vac} 6$ & Model 2 & no / yes & $x$ & no & $\mathcal{N}=0$ & 3 & $\neq 0$ \\
\hline vac 7 & Model 2 & no / yes & $x$ & no & $\mathcal{N}=0$ & 3 & $\neq 0$ \\
\hline vac 8 & Model 2 & no / yes & $\checkmark$ & no & $\mathcal{N}=0$ & 3 & $\neq 0$ \\
\hline vac 9 & Model 3 & yes / yes & $\checkmark$ & yes & $\mathcal{N}=3$ & 6 & $\neq 0$ \\
\hline vac 10 & Model 4 & yes / yes & $\checkmark$ & no & $\mathcal{N}=0$ & 6 & $\neq 0$ \\
\hline $\operatorname{vac} 11$ & Model 4 & yes / yes & $\checkmark$ & no & $\mathcal{N}=1$ & 6 & 0 \\
\hline vac 12 & Model 3 & yes / yes & $\checkmark$ & no & $\mathcal{N}=0$ & 6 & 0 \\
\hline vac 13 & Model 3 & yes / yes & $x$ & no & $\mathcal{N}=0$ & 6 & 0 \\
\hline vac 14 & Model 3 & yes / yes & $x$ & no & $\mathcal{N}=0$ & 3 & $\neq 0$ \\
\hline vac 15 & Model 3 & yes / yes & $\times$ & no & $\mathcal{N}=0$ & 3 & $\neq 0$ \\
\hline vac 16 & Model 4 & yes / yes & $x$ & no & $\mathcal{N}=0$ & 3 & $\neq 0$ \\
\hline vac 17 & Model 3 & yes / yes & $x$ & no & $\mathcal{N}=0$ & 3 & $\neq 0$ \\
\hline
\end{tabular}

Table 5. Data associated to the M-theory landscape compatible with KK6 (KKO6) sources preserving $\mathcal{N}=4$ supersymmetry in four dimensions. All the M-theory backgrounds happen to require a non-vanishing torsion class $\widetilde{W}_{1} \neq 0$.

\subsubsection{Including both types of KK6 (KKO6) sources}

The third prime factor in the decomposition of the system (4.3) demands to simultaneously relax the two condtions

$$
\begin{aligned}
& \text { iv) } \quad \omega_{[i j}^{D} \omega_{k] D}{ }^{7} \neq 0 \rightarrow 3 b_{1} a_{2} \neq 0, \\
& \text { vii) } \quad \omega_{[7 i}^{D} \omega_{j] D}{ }^{k} \neq 0 \rightarrow b_{1} c_{3}^{\prime}+2 c_{1} d_{0} \neq 0,
\end{aligned}
$$

but still imposes the rest of the conditions in (3.3) in order to preserve $\mathcal{N}=4$ supersymmetry. The corresponding M-theory backgrounds then simultaneously include D6 $6_{\|}\left(\mathrm{O} 6_{\|}\right)$ as well as $\mathrm{KK} 6_{\|}\left(\mathrm{KKO} 6_{\|}\right)$sources and cannot be interpreted as an ordinary type IIA orientifold.

This last prime factor is also of dimension one and therefore can be solved explicitly. We find a rich structure of $\mathrm{AdS}_{4}$ critical points they all compatible with

$$
2 c_{1}=\tilde{c}_{1}=\lambda,
$$

so that the $U T$ term in the superpotential (2.42) is absent. Up to discrete multiplicities, we now find nine inequivalent M-theory flux vacua labelled as "vac 9" to "vac 17". As in 
the previous case the phenomenological consequences are very diverse and we have moved a detailed discussion of these M-theory backgrounds to appendix B. A summary of the main features of these vacua is also included in table 5 . For these solutions the net charge of localised sources are

$$
N_{\mathrm{O} 6_{\|}}-N_{\mathrm{D} 6_{\|}}=3 b_{1} a_{2}>0 \quad \text { and } \quad N_{\mathrm{KK} 6_{\|}}-N_{\mathrm{KKO}_{\|}}=b_{1} c_{3}^{\prime}+2 c_{1} d_{0}<0,
$$

requiring the presence of $\mathrm{O} 6 \|^{-}$and $\mathrm{KKO} 6_{\|}$-planes in the backgrounds. Moduli fields can also be fully stabilised at supersymmetric and non-supersymmetric vacua for some of these M-theory backgrounds but, generically, instabilities happen to occur more often.

\subsection{Monopoles and duality orbits of $\mathcal{N}=4$ gaugings}

The results in the previous section have shown that M-theory backgrounds including KK6 (KKO6) monopoles lead to moduli stabilisation in the effective STU-models. Now we will investigate the $\mathcal{N}=4$ gaugings underlying such M-theory backgrounds with sources.

Let us start by recalling the $[\mathrm{SU}(1,1) / \mathrm{U}(1)]^{3}$ duality transformation in (4.1) needed to bring a given moduli configuration to the origin, namely,

$$
S \rightarrow \lambda_{S} S+\Delta_{S}, \quad T \rightarrow \lambda_{T} T+\Delta_{T}, \quad U \rightarrow \lambda_{U} U+\Delta_{U} .
$$

The action of applying (4.21) to the M-theory superpotential in (2.42) has the effect of redefining the flux background (moduli couplings) in the following way

$$
\begin{aligned}
& a_{0} \rightarrow a_{0}+3 \Delta_{U}\left(b_{1} \Delta_{S}+C_{1} \Delta_{T}-a_{1}\right)+3 a_{2} \Delta_{U}^{2}-b_{0} \Delta_{S}+3 \Delta_{T}\left(c_{0}-c_{3}^{\prime} \Delta_{T}-d_{0} \Delta_{S}\right) \\
& a_{1} \rightarrow \lambda_{U}\left(a_{1}-2 a_{2} \Delta_{U}-b_{1} \Delta_{S}-C_{1} \Delta_{T}\right), \quad c_{0} \rightarrow \lambda_{T}\left(c_{0}+C_{1} \Delta_{U}-2 c_{3}^{\prime} \Delta_{T}-d_{0} \Delta_{S}\right) \\
& b_{0} \rightarrow \lambda_{S}\left(b_{0}-3 b_{1} \Delta_{U}+3 d_{0} \Delta_{T}\right), \quad a_{2} \rightarrow \lambda_{U}^{2} a_{2}, \quad b_{1} \rightarrow \lambda_{S} \lambda_{U} b_{1}, \quad C_{1} \rightarrow \lambda_{T} \lambda_{U} C_{1} \\
& c_{3}^{\prime} \rightarrow \lambda_{T}^{2} c_{3}^{\prime}, \quad d_{0} \rightarrow \lambda_{S} \lambda_{T} d_{0},
\end{aligned}
$$

with $C_{1}=\left(2 c_{1}-\tilde{c}_{1}\right)$. Therefore two different M-theory flux vacua among those found in the previous section can be viewed as critical points of the same supergravity model one of them this time moved outside the origin — if their corresponding M-theory flux backgrounds are connected via the transformations (4.22). In other words, if they belong to the same $[\mathrm{SU}(1,1) / \mathrm{U}(1)]^{3}$-duality orbit of flux backgrounds.

It is then easy to check ${ }^{13}$ that the 17 different vacua we found in the previous section belong to uniquely four duality orbits of flux backgrounds. More concretely they group as

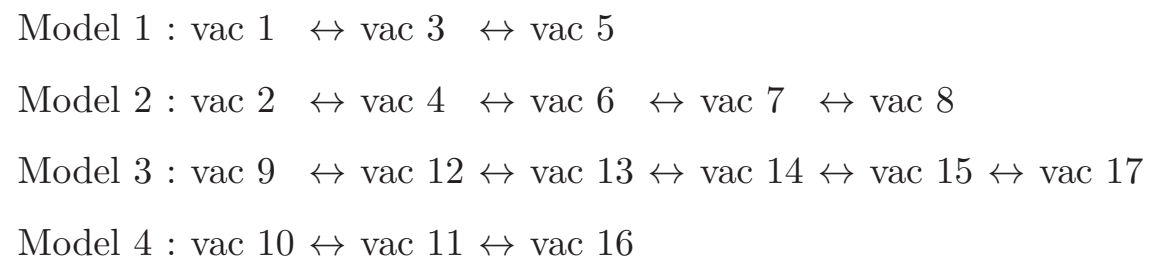

where we have identified one duality orbit with one supergravity model. The $\mathcal{N}=4$ gauging underlying each of the four inequivalent STU-models can be computed by looking

\footnotetext{
${ }^{13}$ Notice that the overall scaling parameter $\lambda$ might be different (and generically will be) in two flux backgrounds related by the transformations (4.22).
} 
at any of the orbit representatives. We decide to select the first representative in each of the models. They are given by

Model 1: $a_{0}=-3 \lambda, \quad a_{1}=b_{0}=c_{0}=0, \quad b_{1}=-d_{0}=\lambda$,

Model 2: $a_{0}=3 \lambda, \quad a_{1}=b_{0}=c_{0}=0, \quad b_{1}=-d_{0}=\lambda$,

Model 3: $a_{0}=-3 \lambda, \quad a_{1}=b_{0}=c_{0}=0, \quad b_{1}=-d_{0}=\lambda, \quad a_{2}=-c_{3}^{\prime}=\frac{\lambda}{2}$,

Model $4: \quad a_{0}=3 \lambda, \quad a_{1}=b_{0}=c_{0}=0, \quad b_{1}=-d_{0}=\lambda, \quad a_{2}=-c_{3}^{\prime}=\frac{\lambda}{2}$,

together with (4.16) for Model 1 and 2 as well as (4.19) for Model 3 and 4. A detailed description of these M-theory backgrounds is collected in the appendices. Remarkably they require $a_{1}=b_{0}=c_{0}=0$ implying a vanishing flux $G_{(4)}=0$ (see table 1 ) as well as a non-vanishing $G_{(7)} \neq 0$. The result is then an M-theory superpotential (2.6) of the form

$$
W_{\mathrm{M} \text {-theory }}=\frac{1}{4} \int_{X_{7}} G_{(7)}+\frac{1}{8} \int_{X_{7}}\left(A_{(3)}+i \Phi_{(3)}\right) \wedge d\left(A_{(3)}+i \Phi_{(3)}\right),
$$

uniquely induced by metric $\omega$ and $G_{(7)}$ background fluxes. The underlying $\mathcal{N}=4$ gauging turns out to have a very simple algebra structure as we will investigate now.

The four inequivalent STU-models can be simultaneously explored by considering the gauge algebra $G_{0} \subset \mathrm{SL}(2) \times \mathrm{SO}(6,6)$ induced by the set of seven fluxes

$$
\begin{array}{ll}
a_{0}=-f_{+}{ }^{a b c}, & a_{2}=-f_{+}{ }^{a j k}, \\
b_{1}=f_{-}{ }^{i b c}, & d_{0}=f_{+}{ }^{b c}{ }^{b c},
\end{array}
$$

where the upper line contains electric fluxes $(\alpha=+)$ and the lower line magnetic ones $(\alpha=-)$. In order to analyse the structure of the gauge brackets in (3.4), we use again the splitting of the $\mathrm{SO}(6,6)$ light-cone index $M=\left(a,{ }_{i},{ }^{a},{ }^{i}\right)$ in the generators $T_{\alpha M}$

$$
\begin{aligned}
& T_{+a} \equiv Z_{a}, \quad T_{+i} \equiv Z_{i}, \quad T_{+}{ }^{a} \equiv X^{a}, \quad T_{+}{ }^{i} \equiv X^{i}, \\
& T_{-a} \equiv \bar{Z}_{a}, \quad T_{-i} \equiv \bar{Z}_{i}, \quad T_{-}{ }^{a} \equiv \bar{X}^{a}, \quad T_{-}{ }^{i} \equiv \bar{X}^{i} .
\end{aligned}
$$

Using this decomposition, the antisymmetry of the brackets in (3.4) determines the magnetic generators in terms of the electric ones so that only an independent twelve-dimensional algebra is gauged. One obtains

$$
\bar{Z}_{a}=0, \quad \bar{Z}_{i}=\frac{c_{1} d_{0}-b_{1} c_{3}^{\prime}}{c_{1}^{2}+a_{2} c_{3}^{\prime}} Z_{a}, \quad \bar{X}^{a}=\frac{b_{1}}{\tilde{c}_{1}} Z_{i}+\frac{d_{0}}{\tilde{c}_{1}} X^{i}, \quad \bar{X}^{i}=\frac{b_{1} c_{1}+a_{2} d_{0}}{c_{1}^{2}+a_{2} c_{3}^{\prime}} Z_{a} .
$$

According to the Levi decomposition of finite dimensional real lie algebras, we find that $G_{0}=G_{\text {semi }} \ltimes G_{\text {solv }}$ with a 3 -dimensional semisimple piece and a 9-dimensional solvable piece. The generators $\left\{Z_{i}, X^{i} ; Z_{a}\right\}$ span the solvable (actually nilpotent) ideal with nonvanishing brackets

$$
\left[Z_{i}, Z_{j}\right]=c_{3}^{\prime} Z_{c}, \quad\left[Z_{i}, X^{j}\right]=c_{1} Z_{c}, \quad\left[X^{i}, X^{j}\right]=-a_{2} Z_{c}
$$


which can be understood as $G_{\text {solv }}=\mathrm{U}(1)^{6} \ltimes \mathrm{U}(1)^{3}$. The mixed brackets between the solvable and the semisimple pieces read

$$
\left[Z_{a}, X^{b}\right]=\tilde{c}_{1} Z_{c}, \quad\left[Z_{i}, X^{b}\right]=c_{1} Z_{k}+c_{3}^{\prime} X^{k}, \quad\left[X^{i}, X^{b}\right]=-a_{2} Z_{k}+c_{1} X^{k},
$$

whereas the non-vanishing commutators between generators $X^{a}$ in the semisimple piece are given by

$$
\left[X^{a}, X^{b}\right]=\tilde{c}_{1} X^{c}-a_{0} Z_{c} .
$$

The full 12-dimensional $\mathcal{N}=4$ gauging thus corresponds to a gauge group

$$
G_{0}=\mathrm{SO}(3) \ltimes \operatorname{Nil}_{9}(2),
$$

with $\mathrm{Nil}_{9}(2)$ being a 9-dimensional $\mathrm{U}(1)^{6} \ltimes \mathrm{U}(1)^{3}$ nilpotent ideal of order two (three steps) with lower central series

$$
\left\{Z_{i}, X^{i}, Z_{a}\right\} \supset\left\{Z_{a}\right\} \supset 0 .
$$

The different values of the fluxes in the four disconnected STU-models only determine how the semisimple products are specifically realised but do not modify the identification of the full group as $G_{0}=\mathrm{SO}(3) \ltimes \mathrm{Nil}_{9}(2)$. This gauge group (with a different realisation in terms of brackets) has also appeared in twisted reductions of massive type IIA strings [8, 9]. However, as we already emphasised, those massive type IIA backgrounds cannot be obtained from our M-theory reductions due to the lack of the Romans mass parameter. ${ }^{14}$

\subsection{Overview of M-theory backgrounds with monopoles}

Let us summarise the set of M-theory flux vacua we have obtained when including monopoles in the background and also discuss their main features. Turning on KK6 (KKO6) monopoles compatible with $\mathcal{N}=4$ supersymmetry of the effective action, we have found three different situations:

1) In the first situation the M-theory background is compatible with having only KKO6planes admitting an interpretation in terms of $\mathrm{O}_{\|}$-planes in a type IIA orientifold incarnation of the effective STU-model. The effective flux model is a no-scale supergravity and corresponds to the "vac 0" solution in table 5. This scenario was discussed in detail in section 4.3.1.

2) The second situation involves M-theory backgrounds compatible with having only KKO6 ${ }_{\|}$-planes, hence lacking a type IIA interpretation in terms of an ordinary STU orientifold model. There are 8 different backgrounds lying inside two different duality orbits of theories (inequivalent superpotentials) after making use of duality transformations in the effective action. Full moduli stabilisation can be achieved in this type of backgrounds producing supersymmetric $(\mathcal{N}=1,3)$ as well as non-supersymmetric

\footnotetext{
${ }^{14}$ The Romans mass parameter $a_{3}$ generates the only cubic coupling $-a_{3} U^{3}$ in the IIA superpotential (2.43). This term cannot be removed by applying the STU duality transformations in (4.21), hence forcing the massive IIA backgrounds to lie in a different duality orbit of STU-models than the M-theory backgrounds.
} 
and stable $\mathrm{AdS}_{4}$ vacua. An analysis of the $G_{2}$-structure underlying these solutions reveals the existence of one supersymmetric $(\mathcal{N}=1)$ and two non-supersymmetric backgrounds with weak $G_{2}$ holonomy. The rest correspond to $G_{2}$-structures where torsion classes both in the $\mathbf{1}\left(\widetilde{W}_{1}\right)$ and the $\mathbf{2 7}\left(\widetilde{W}_{27}\right)$ are activated. In all the M-theory backgrounds within this category the $\mathcal{N}=4$ gauging $G_{0}=\mathrm{SO}(3) \ltimes \mathrm{Nil}_{9}(2)$ is broken to a $G_{\text {res }}=\mathrm{SO}(3)$ subgroup at the vacuum. In addition, some unstable solutions also exist. The results are summarised in the second block of table 5 encompassing solutions from vac 1 to vac 8 .

3) The third and last situation corresponds to M-theory backgrounds compatible with having simultaneously $\mathrm{O}_{\|}$-planes as well as KKO6 ${ }_{\|}$-planes. Due to the necessity of the latter, no type IIA interpretation in terms of ordinary STU orientifold models is possible either. There are 9 different M-theory backgrounds — from vac 9 to vac 17 - lying inside two duality orbits of inequivalent theories also with an $\mathcal{N}=4$ gauging $G_{0}=\mathrm{SO}(3) \ltimes \mathrm{Nil}_{9}(2)$, although it has a slightly different realisation in terms of gauge brackets (see discussion in section 4.3). One remarkable consequence of having the two types of sources is that the residual symmetry group gets enhanced to $G_{\text {res }}=\mathrm{SO}(3) \ltimes \mathrm{U}(1)^{3}$ at the supersymmetric $(\mathcal{N}=1,3)$ solutions as well as at the three non-supersymmetric solutions vac 10 (stable), vac 12 (stable) and vac 13 (unstable) in table 5. The rest of the solutions in this category turn out to be non-supersymmetric, unstable and preserve $G_{\text {res }}=\mathrm{SO}(3)$. Concerning the $G_{2^{-}}$ structure of these backgrounds, the situation is similar to the previous case: there is one supersymmetric $(\mathcal{N}=1)$ and two non-supersymmetric M-theory backgrounds with weak $G_{2}$ holonomy. The rest of the solutions activate $\widetilde{W}_{1}$ as well as $\widetilde{W}_{27}$. These results are collected in the third block of table 5 .

The above set of M-theory backgrounds has been obtained after relaxing the ordinary Scherk-Schwarz conditions (3.1) in a way compatible with $\mathcal{N}=4$ supersymmetry in the effective action. One could completely forget about the entire set of conditions in (3.1) if permitting all the types of sources in table 4 to be present in the background. The resulting theory would then just preserve $\mathcal{N}=1$ supersymmetry. In this case new solutions might (and generically will) appear involving more complex configurations of sources in higher dimensions and consequently more elaborated flux backgrounds and superpotentials in four dimensions. In addition, due to the rich structure of moduli powers in the scalar potential (see section 3.4), one might hope for the existence of de Sitter solutions in these M-theory scenarios. However a no-go theorem forbidding the existence of such solutions can be derived along the lines of ref. [36] using the M-theory universal moduli [45]. Even if charting the landscape of such unrestricted M-theory configurations could be too ambitious, one could still restrict the scan to solutions preserving weak $G_{2}$ holonomy or some other restricted structure of torsion classes. For the sake of simplicity, we have just restricted ourselves in this work to M-theory backgrounds preserving $\mathcal{N}=4$ supersymmetry in four dimensions. 


\section{Summary and final remarks}

In this paper we have investigated M-theory reductions on $G_{2}$-manifolds in the presence of gauge and metric background fluxes as well as KK6 (KKO6) sources from a purely fourdimensional point of view. We have done it in terms of the $\mathcal{N}=1$ effective STU-models describing truncations of $\mathcal{N}=4$ gauged supergravity on the basis of the embedding tensor formalism $[3,35]$.

In the first part of the paper we investigated the interplay between the conditions (3.1) required by an ordinary Scherk-Schwarz reduction and the consistency relations (3.6) and (3.8) imposed by $\mathcal{N}=4$ and $\mathcal{N}=8$ supersymmetry on the corresponding gauged supergravity. The outcome was that while demanding $\mathcal{N}=8$ supersymmetry in the effective action amounts to imposing the entire set of conditions in (3.1), requiring only $\mathcal{N}=4$ allows for a relaxation of some of the Scherk-Schwarz conditions. More concretely, the conditions $i v$ ) and vii) in the list of (3.3). The non-vanishing of these two conditions was respectively linked to the presence of $\mathrm{D} 6_{\|}\left(\mathrm{O} 6_{\|}\right)$and $\mathrm{KK} 6_{\|}\left(\mathrm{KKO} 6_{\|}\right)$sources in the background (see table 4) and also to the activation of the genuine M-theory fluxes $\left(c_{3}^{\prime}, d_{0}\right)$ in the superpotential (1.2) which had no counterpart in the type IIA orientifold constructions of refs $[4,8,9,30]$. The novel $\mathrm{KK}_{\|}\left(\mathrm{KKO}_{\|}\right)$sources were found to induce new universal moduli powers in the scalar potential (3.13) thus opening new possibilities for moduli stabilisation in M-theory flux models.

In the second part of the paper we performed a systematic and exhaustive study of M-theory flux vacua by combining the use of dualities in the STU-models with algebraic geometry tools available in the computer algebra system SINGULAR. We proved that full moduli stabilisation can be achieved in $\mathcal{N}=4$ flux models coming from M-theory provided KKO6-planes are included as background sources (see table 5 and section 4.5 for a summary of the results). The underlying $\mathcal{N}=4$ gauging is unique and identified with $G_{0}=\mathrm{SO}(3) \ltimes \mathrm{Nil}_{9}(2)$. Moreover, we also showed that these models correspond to genuine M-theory backgrounds which do not admit an interpretation in terms of regular type IIA orientifold constructions. In the latter, moduli stabilisation seems to demand a non-vanishing Romans mass parameter [10] and therefore a deformation already in higher dimensions $[8,9]$. This deformation parameter does not appear from M-theory upon ordinary dimensional reduction so, in the M-theory backgrounds we have found here, full moduli stabilisation is achieved from a massless theory in higher dimension. Moreover a background flux for the $G_{(7)}$ form - $a_{0}$ parameter in (2.42) — seems to be mandatory in this case, thus playing a similar role as the Romans mass for moduli stabilisation in a type IIA context but having a neat "field strength" interpretation in higher dimensions as the dual of a purely external $G_{(4)}$ flux $[12,46]$.

Finally we want to stress once more the four-dimensional, bottom-up approach adopted in this paper which justifies to adopt the ET formalism to analyse the effect of the particular M-theory fluxes without a type IIA interpretation, namely, M-theory fluxes becoming non-geometric in the IIA picture. While focusing on four-dimensional solutions has interest for obvious reason, one may feel concerned with the actual existence of a ten-dimensional type IIA interpretation of the flux vacua we found here. The ultimate connection should be 
between strings/M-theory and four-dimensional physics, and insisting on an intermediate ten-dimensional field theory step could appear somewhat artificial. ${ }^{15}$ On the other hand, because of the 16 supercharges they preserve, it would be interesting to explore potential realisations of the gauged supergravities studied here as eleven-dimensional 1/2-BPS backgrounds. We find the reach structure of M-theory flux vacua presented in this work an additional motivation for pursuing this goal. We hope to come back to this in the future.

\section{Acknowledgments}

We want to thank Ulf Danielsson, Giuseppe Dibitetto and Diederik Roest for interesting discussions and specially Giuseppe Dibitetto for the careful reading of an early draft of this paper. The work of the authors is supported by the Swiss National Science Foundation.

\section{A Backgrounds with only $\mathrm{KK} 6_{\|}\left(\mathrm{KKO} 6_{\|}\right)$}

In this first appendix we present the detailed analysis of the eight M-theory backgrounds in section 4.3 .2 which include only $\mathrm{KK} 6(\mathrm{KKO} 6) \rightarrow \mathrm{KK} 6_{\|}\left(\mathrm{KKO}_{\|}\right)$sources. They all are compatible with the flux condition (4.16). We present the associated background fluxes, M-theory superpotential, vacuum energy $V_{0}$, normalised ${ }^{16}$ mass spectra for scalars and vectors and preserved supersymmetry at each of the M-theory flux vacua.

Vacuum 1. This solution corresponds to the flux configuration

$$
a_{0}=-3 \lambda, \quad a_{1}=b_{0}=c_{0}=0, \quad b_{1}=-d_{0}=\lambda,
$$

which implies a superpotential (2.42) of the form

$$
W_{\mathrm{M}-\text { Theory }}^{\text {(iso) }}=3 \lambda(S T+S U+T U-1) .
$$

The value of the potential evaluated at this critical points is $V_{0}=-3 \lambda^{2} / 8$ corresponding to an $\mathrm{AdS}_{4}$ solution. The scalar masses turn out to be

$$
m^{2} L^{2}=18(\times 1), \quad 10(\times 6), 4(\times 7),-2(\times 5), \quad 0(\times 19),
$$

not displaying instabilities but displaying ten flat directions not associated to Goldstone bosons. The vector masses read

$$
m^{2} L^{2}=12(\times 3), 6(\times 3), 2(\times 3), 0(\times 3),
$$

where the three massless vectors are associated to the $\mathrm{SO}(3)$ residual symmetry preserved by the STU models. The computation of the gravitini mass matrix (4.4) reveals $\mathcal{N}=3$ preserved supersymmetry.

\footnotetext{
${ }^{15}$ Four-dimensional gauged supergravities have been shown to capture the dynamics of asymmetric orbifold constructions for which a ten-dimensional "geometry" does not even exist due to the difference between the left $X_{L}$ and right $X_{R}$ sectors. Further interesting connections to non-geometric flux backgrounds have also been established in refs [47-49].

${ }^{16}$ We normalise the masses with respect to the $\mathrm{AdS}_{4}$ radius $L=\sqrt{-3 / V_{0}}$ so that perturbative stability requires tachyons to satisfy the Breitenlohner-Freedman (BF) [50] bound $m^{2} L^{2} \geq-9 / 4$.
} 
Vacuum 2. This solution simply changes the sign of $a_{0}$ compared to (A.1)

$$
a_{0}=3 \lambda, \quad a_{1}=b_{0}=c_{0}=0, \quad b_{1}=-d_{0}=\lambda,
$$

so the superpotential now reads

$$
W_{\mathrm{M}-\text { Theory }}^{(\mathrm{iso})}=3 \lambda(S T+S U+T U+1) \text {. }
$$

At this critical point the value of the energy is also $V_{0}=-3 \lambda^{2} / 8$. The computation of the scalar masses

$$
m^{2} L^{2}=18(\times 2), \quad 10(\times 5), \quad 4(\times 6),-2(\times 7), \quad 0(\times 18),
$$

gives different multiplicities compared to (A.3) and nine flat directions. The vector masses are given by

$$
m^{2} L^{2}=12(\times 3), \quad 6(\times 3), 2(\times 3), \quad 0(\times 3),
$$

coinciding with those in (A.4). However, the computation of the gravitini mass matrix (4.4) shows that supersymmetry is completely broken at this solution.

Vacuum 3. This solution is compatible with the family of flux parameters

$$
a_{0}=-\frac{9}{5} \lambda, \quad a_{1}=b_{0}=c_{0}=0, \quad b_{1}=-d_{0}=\frac{1}{5} \lambda,
$$

so this time the superpotential is given by

$$
W_{\mathrm{M}-\text { Theory }}^{\text {(iso) }}=\frac{3}{5} \lambda(S T+S U+5 T U-3) .
$$

The value of the vacuum energy at this solution is $V_{0}=-27 \lambda^{2} / 200$. Computation of the scalar masses we find

$$
\begin{aligned}
m^{2} L^{2}= & \frac{190}{9}(\times 5), \quad 18(\times 1), \frac{112}{9}(\times 5), \frac{70}{9}(\times 1), \quad \frac{52}{9}(\times 5), \frac{32}{9}(\times 3), \\
& -\frac{20}{9}(\times 1), \quad-2(\times 1), \frac{10}{9}(\times 6), \quad-\frac{8}{9}(\times 1), 0(\times 9),
\end{aligned}
$$

so there are neither instabilities (tachyons satisfy the BF bound) nor flat directions at this vacuum (the nine massless scalars correspond to Goldstone bosons). For the vector masses we obtain

$$
m^{2} L^{2}=12(\times 3), 6(\times 3), \quad \frac{50}{9}(\times 3), 0(\times 3),
$$

displaying three massless vectors associated to the $\mathrm{SO}(3)$ residual symmetry. Supersymmetry is completely broken at this solution. 
Vacuum 4. This solution is very similar to (A.9). Again there is a sign flip for the $a_{0}$ flux parameter. The background fluxes is given by

$$
a_{0}=\frac{9}{5} \lambda, \quad a_{1}=b_{0}=c_{0}=0, \quad b_{1}=-d_{0}=\frac{1}{5} \lambda,
$$

so the superpotential reads

$$
W_{\mathrm{M}-\text { Theory }}^{\text {(iso) }}=\frac{3}{5} \lambda(S T+S U+5 T U+3),
$$

and the vacuum energy at this solution is also $V_{0}=-27 \lambda^{2} / 200$. The mass spectrum for the scalars turns out to be

$$
\begin{aligned}
m^{2} L^{2}= & \frac{190}{9}(\times 5), \quad 18(\times 1), \frac{112}{9}(\times 5), \quad 10(\times 1), \frac{52}{9}(\times 5), \frac{32}{9}(\times 3), \\
& -\frac{20}{9}(\times 2), \quad \frac{10}{9}(\times 5), \quad-\frac{8}{9}(\times 2), \quad 0(\times 9),
\end{aligned}
$$

again featuring neither instabilities nor flat directions. The spectrum of vector masses is

$$
m^{2} L^{2}=12(\times 3), \quad 6(\times 3), \frac{50}{9}(\times 3), \quad 0(\times 3),
$$

and coincides with that of (A.12). Substituting the values of the fluxes into the gravitini mass matrix (4.4), this solution happens to preserve $\mathcal{N}=1$ supersymmetry.

Vacuum 5. This solution corresponds to background fluxes of the form

$$
a_{0}=-\frac{6}{5} \lambda, \quad a_{1}=b_{0}=-c_{0}=\frac{3}{5} \lambda, \quad b_{1}=-d_{0}=\frac{1}{5} \lambda,
$$

and induces the flux superpotential

$$
W_{\mathrm{M}-\mathrm{Theory}}^{(\text {iso })}=\frac{3}{5} \lambda(-S-3 T-3 U+S T+S U+5 T U-2) .
$$

It produces a vacuum energy $V_{0}=-9 \lambda^{2} / 80$. We find the following mass spectrum for the scalars

$$
\begin{aligned}
m^{2} L^{2}= & \frac{76}{3}(\times 5), 18(\times 1), \quad \frac{64}{3}(\times 5), \frac{28}{3}(\times 1), \frac{20}{3}(\times 3), 6(\times 1), \\
& \frac{16}{3}(\times 5), \quad \frac{4}{3}(\times 6),-\frac{2}{3}(\times 2), 0(\times 9),
\end{aligned}
$$

which does not present neither instabilities nor flat directions. The vector mass spectrum is given by

$$
m^{2} L^{2}=12(\times 6), \frac{20}{3}(\times 3), 0(\times 3),
$$

containing the three $\mathrm{SO}(3)$ massless vectors. The gravitini mass matrix shows that supersymmetry is completely broken at this solution. 
Vacuum 6. This solution is generated by a flux background given by

$$
a_{0}=\lambda, \quad a_{1}=c_{0}=-\frac{1}{2} \sqrt{\frac{1}{6}(31+3 \sqrt{57})} \lambda, \quad b_{0}=0, \quad b_{1}=-d_{0}=\frac{1}{12}(9+\sqrt{57}) \lambda,
$$

and the associated superpotential lacks the linear term on the dilaton modulus $S$. The vacuum energy at this solution is $V_{0}=-\frac{1}{64}(13+\sqrt{57}) \lambda^{2}$. Computing the masses for the scalars we obtain

$$
\begin{aligned}
m^{2} L^{2}= & 27.976(\times 1), \quad 18(\times 1), \quad 16.921(\times 5), \quad 13.771(\times 1), \\
& 10.107(\times 1), \quad 10.083(\times 5), \quad 9.221(\times 1), \quad 5.557(\times 3), \\
& -3.961(\times 5), \quad-2.648(\times 1), \quad 2.086(\times 5), \quad 0(\times 9) .
\end{aligned}
$$

This time there are tachyon masses violating the BF bound, thus rendering this solution unstable. Still there are no flat directions as the nine massless scalars correspond to Goldstone modes. The computation of the vector masses yields

$$
m^{2} L^{2}=12(\times 3), \quad \frac{1}{7}(97-\sqrt{57})(\times 3), \quad \frac{1}{7}(71+\sqrt{57})(\times 3), \quad 0(\times 3),
$$

containing the three massless vectors of the $\mathrm{SO}(3)$ residual symmetry. This is a nonsupersymmetric and unstable solution.

Vacuum 7. This solution is induced by a family of fluxes of the form

$$
a_{0}=\lambda, \quad a_{1}=c_{0}=-\frac{1}{2} \sqrt{\frac{1}{6}(31-3 \sqrt{57})} \lambda, \quad b_{0}=0, \quad b_{1}=-d_{0}=\frac{1}{12}(9-\sqrt{57}) \lambda
$$

then being very similar to the previous solution (A.21) and lacking also the linear term on $S$. The vacuum energy is nevertheless different $V_{0}=-\frac{1}{64}(13-\sqrt{57}) \lambda^{2}$. The spectrum of scalar masses is given by

$$
\begin{aligned}
m^{2} L^{2}= & 31.857(\times 5), \quad 18(\times 1), \quad 30.364(\times 5), \quad 12.686(\times 1), \\
& 9.871(\times 3), \quad 7.064(\times 1), \quad 6.564(\times 5), \quad 3.799(\times 5), \\
& -3.486(\times 1), \quad-0.678(\times 1), \quad-0.300(\times 1), \quad 0(\times 9),
\end{aligned}
$$

and features one tachyon mass violating the BF bound. The solution is then unstable and does not contain flat directions. The set of vector masses reads

$$
m^{2} L^{2}=12(\times 3), \frac{1}{7}(97+\sqrt{57})(\times 3), \quad \frac{1}{7}(71-\sqrt{57})(\times 3), \quad 0(\times 3),
$$

being then similar to (A.23) and, as usual, showing the three massless vectors of the $\mathrm{SO}(3)$ symmetry. Computing the gravitini mass matrix (4.4) one finds that this solution is nonsupersymmetric. 
Vacuum 8. The last solution of this section is associated to the most complex family of background fluxes

$$
\begin{aligned}
& a_{0}=\frac{5}{4} \lambda, \quad a_{1}=\frac{1}{24}(\sqrt{15}-3 \sqrt{35}) \lambda, \quad b_{0}=-\frac{\sqrt{15}}{4} \lambda, \\
& b_{1}=\frac{1}{24}(9-\sqrt{21}) \lambda, \quad c_{0}=-\frac{1}{4} \sqrt{\frac{5}{6}(11+\sqrt{21})} \lambda, \quad d_{0}=-\frac{1}{24}(9+\sqrt{21}) \lambda .
\end{aligned}
$$

The flux-induced superpotential consists of the same moduli couplings as (A.18) this time specified by (A.21), and produces a vacuum energy $V_{0}=-5 \lambda^{2} / 32$. The scalar mass spectrum is given by ${ }^{17}$

$$
\begin{aligned}
m^{2} L^{2}= & 20.955(\times 5), \quad 18(\times 1), \quad 14.341(\times 5), \quad 14.181(\times 1), \\
& 3.986(\times 5), \quad 2.539(\times 1),-1.150(\times 1), \quad-1.120(\times 1), \\
& \frac{24}{5}(\times 3), \quad-0.882(\times 5), \quad 8.350(\times 1), \quad 0(\times 9),
\end{aligned}
$$

which does not contain instabilities or flat directions. The masses of the vectors are

$$
m^{2} L^{2}=12(\times 3), \quad \frac{2}{5}(21 \pm \sqrt{46})(\times 3), \quad 0(\times 3),
$$

featuring the three massless vectors of the $\mathrm{SO}(3)$ residual symmetry. This solution is non-supersymmetric and perturbatively stable.

\section{B Backgrounds with $\mathrm{KK}_{\|}\left(\mathrm{KKO} 6_{\|}\right) \& \mathrm{D} 6_{\|}\left(\mathrm{O} 6_{\|}\right)$}

In this second appendix we present the detailed analysis of the nine M-theory backgrounds in section 4.3.3 which include both $\mathrm{KK} 6(\mathrm{KKO} 6) \rightarrow \mathrm{KK}_{\|}\left(\mathrm{KKO}_{\|}\right)$as well as KK6 (KKO6) $\rightarrow \mathrm{D}_{\|}\left(\mathrm{O} 6_{\|}\right)$sources. They all are this time compatible with the flux condition (4.19). As in the previous appendix, we present the associated background fluxes, M-theory superpotential, vacuum energy $V_{0}$, normalised mass spectra for scalars and vectors and preserved supersymmetry at each of the M-theory flux vacua.

Vacuum 9. The first of these solutions is induced by a flux background

$$
a_{0}=-3 \lambda, \quad a_{1}=b_{0}=c_{0}=0, \quad a_{2}=-c_{3}^{\prime}=\frac{1}{2} \lambda, \quad b_{1}=-d_{0}=\lambda,
$$

which implies a superpotential (2.42) of the form

$$
W_{\mathrm{M} \text {-Theory }}^{\text {(iso) }}=3 \lambda\left(S T+S U+\frac{1}{2} T^{2}+\frac{1}{2} U^{2}-1\right) .
$$

The value of the potential evaluated at this critical point is $V_{0}=-3 \lambda^{2} / 8$. The set of scalar masses reads

$$
m^{2} L^{2}=18(\times 1), \quad 10(\times 6), 4(\times 6),-2(\times 18), \quad 0(\times 7),
$$

\footnotetext{
${ }^{17}$ For the sake of clarity we display the numerical value of irrational numbers.
} 
not displaying instabilities but containing one flat direction. The set of vector masses is given by

$$
m^{2} L^{2}=12(\times 3), 6(\times 3), 0(\times 6),
$$

where the six massless vectors are associated to an enhancement of the $\mathrm{SO}(3)$ residual symmetry preserved by the STU models. Computing the gravitini mass matrix (4.4) shows that this solution preserves $\mathcal{N}=3$ supersymmetry. Notice the similarities with the vacuum 1 discussed in the previous appendix.

Vacuum 10. This solution is generated from a flux background like (B.1) after flipping the sign of the $a_{0}$ flux parameter. This is

$$
a_{0}=3 \lambda, \quad a_{1}=b_{0}=c_{0}=0, \quad a_{2}=-c_{3}^{\prime}=\frac{1}{2} \lambda, \quad b_{1}=-d_{0}=\lambda,
$$

and induces a superpotential

$$
W_{\mathrm{M} \text {-Theory }}^{(\text {iso) }}=3 \lambda\left(S T+S U+\frac{1}{2} T^{2}+\frac{1}{2} U^{2}+1\right) .
$$

At this critical point, the value of the vacuum energy is also $V_{0}=-3 \lambda^{2} / 8$. Computing the spectrum of scalar masses we find

$$
m^{2} L^{2}=18(\times 2), 4(\times 15),-2(\times 15), 0(\times 6),
$$

not displaying instabilities and without flat directions. The mass spectrum for the vectors reads

$$
m^{2} L^{2}=12(\times 3), 6(\times 3), 0(\times 6),
$$

with six massless vectors being again associated to an enhancement of the $\mathrm{SO}(3)$ residual symmetry. This solution turns out to be non-supersymmetric and perturbatively stable.

Vacuum 11. This solution is obtained from the flux background

$$
a_{0}=\frac{9}{5} \lambda, \quad a_{1}=b_{0}=c_{0}=0, \quad a_{2}=-c_{3}^{\prime}=\frac{1}{2} \lambda, \quad b_{1}=-d_{0}=\frac{1}{5} \lambda,
$$

which induces the flux superpotential

$$
W_{\mathrm{M} \text {-Theory }}^{\text {(iso) }}=\frac{3}{5} \lambda\left(S T+S U+\frac{5}{2} T^{2}+\frac{5}{2} U^{2}+3\right) .
$$

The value of the vacuum energy is $V_{0}=-27 \lambda^{2} / 200$. The scalar mass spectrum at this critical point consists of

$$
\begin{aligned}
m^{2} L^{2}= & 10(\times 1), \quad 18(\times 1), \frac{22}{9}(\times 9), \frac{70}{9}(\times 9), \frac{52}{9}(\times 5), \\
& -\frac{20}{9}(\times 1), \frac{10}{9}(\times 5),-\frac{8}{9}(\times 1), 0(\times 6),
\end{aligned}
$$

thus not containing instabilities nor flat directions. The set of vector masses is given by

$$
m^{2} L^{2}=12(\times 3), \quad 6(\times 3), \quad 0(\times 6),
$$

showing six massless vectors associated to the residual symmetry. This solution preserves $\mathcal{N}=1$ supersymmetry. 
Vacuum 12. This solution is related to that in (B.9) again by a sign flip of the $a_{0}$ flux. The flux background reads

$$
a_{0}=-\frac{9}{5} \lambda, \quad a_{1}=b_{0}=c_{0}=0, \quad a_{2}=-c_{3}^{\prime}=\frac{1}{2} \lambda, \quad b_{1}=-d_{0}=\frac{1}{5} \lambda
$$

and induces the moduli superpotential

$$
W_{\mathrm{M}-\text { Theory }}^{\text {(iso) }}=\frac{3}{5} \lambda\left(S T+S U+\frac{5}{2} T^{2}+\frac{5}{2} U^{2}-3\right) .
$$

As for the previous case, the vacuum energy is $V_{0}=-27 \lambda^{2} / 200$. The computation of the scalar masses gives

$$
\begin{aligned}
m^{2} L^{2}= & \frac{190}{9}(\times 5), \quad 18(\times 1), \quad \frac{52}{9}(\times 5), \quad \frac{22}{9}(\times 9),-\frac{20}{9}(\times 10), \\
& -2(\times 1), \quad \frac{10}{9}(\times 1), \quad 0(\times 6),
\end{aligned}
$$

so it does not present instabilities or flat directions. The mass spectrum for the vectors consists of

$$
m^{2} L^{2}=12(\times 3), 6(\times 3), 0(\times 6),
$$

featuring the usual six massless vectors associated to the residual symmetry of these solutions. Supersymmetry is completely broken at this solution.

Vacuum 13. This solution corresponds to background fluxes of the form

$$
a_{0}=-\frac{6}{5} \lambda, \quad a_{1}=b_{0}=-c_{0}=\frac{3}{5} \lambda, \quad a_{2}=-c_{3}^{\prime}=\frac{1}{2} \lambda, \quad b_{1}=-d_{0}=\frac{1}{5} \lambda,
$$

and induces the flux superpotential

$$
W_{\mathrm{M}-\text { Theory }}^{(\text {iso) }}=\frac{3}{5} \lambda\left(-S-3 T-3 U+S T+S U+\frac{5}{2} T^{2}+\frac{5}{2} U^{2}-2\right) .
$$

It has a vacuum energy $V_{0}=-9 \lambda^{2} / 80$. The mass spectrum for the scalars is given by

$$
\begin{aligned}
m^{2} L^{2}= & \frac{76}{3}(\times 5), \quad 18(\times 1), \quad \frac{22}{3}(\times 9), \frac{16}{3}(\times 5),-\frac{8}{3}(\times 9), 6(\times 1), \\
& \frac{4}{3}(\times 1), \quad-\frac{2}{3}(\times 1), \quad 0(\times 6),
\end{aligned}
$$

which contains instabilities (modes with $m^{2} L^{2}=-\frac{8}{3}$ ) and has no flat directions. The vector masses are

$$
m^{2} L^{2}=12(\times 6), 0(\times 6),
$$

containing six massless vectors associated to the residual symmetry. The gravitini mass matrix shows that this solution is non-supersymmetric. 
Vacuum 14. This solution is generated by a flux background given by

$$
\begin{aligned}
& a_{0}=-\lambda, \quad a_{1}=c_{0}=-\frac{1}{2} \sqrt{\frac{1}{6}(31+3 \sqrt{57})} \lambda, \quad b_{0}=0, \quad a_{2}=-c_{3}^{\prime}=\frac{1}{2} \lambda, \\
& b_{1}=-d_{0}=\frac{1}{12}(9+\sqrt{57}) \lambda,
\end{aligned}
$$

which is similar to (A.21). The associated superpotential also lacks the linear term on the dilaton modulus $S$. The vacuum energy is $V_{0}=-\frac{1}{64}(13+\sqrt{57}) \lambda^{2}$. We find the scalar mass spectrum

$$
\begin{aligned}
m^{2} L^{2}= & 21.308(\times 1), \quad 18(\times 1), \quad 13.328(\times 1), \quad 13.771(\times 1), \\
& 12.942(\times 5), \quad 12.036(\times 5), 9.221(\times 1), \quad-5.643(\times 1), \\
& -2.693(\times 5), \quad 2.336(\times 3), \quad-0.942(\times 5), \quad 0(\times 9),
\end{aligned}
$$

which displays tachyons violating the $\mathrm{BF}$ bound and has no flat directions. The mass spectrum for the vectors reads

$$
m^{2} L^{2}=\frac{1}{7}(71+\sqrt{57} \pm \sqrt{1186-74 \sqrt{57}})(\times 3), \quad \frac{1}{7}(71+\sqrt{57})(\times 3), \quad 0(\times 3),
$$

containing the three massless vectors of the $\mathrm{SO}(3)$ residual symmetry. This is a nonsupersymmetric and unstable solution.

Vacuum 15. This solution is induced by a family of fluxes of the form

$$
\begin{aligned}
& a_{0}=-\lambda, \quad a_{1}=c_{0}=-\frac{1}{2} \sqrt{\frac{1}{6}(31-3 \sqrt{57})} \lambda, \quad b_{0}=0, \quad a_{2}=-c_{3}^{\prime}=\frac{1}{2} \lambda, \\
& b_{1}=-d_{0}=\frac{1}{12}(9-\sqrt{57}) \lambda,
\end{aligned}
$$

which is similar to (B.21) and produces a vacuum energy $V_{0}=-\frac{1}{64}(13-\sqrt{57}) \lambda^{2}$. The spectrum of scalar masses is given by

$$
\begin{aligned}
m^{2} L^{2}= & 31.235(\times 5), \quad 18(\times 1), \quad 13.936(\times 5), \quad 11.458(\times 1), \\
& 8.807(\times 3), \quad 7.064(\times 1), \quad 3.994(\times 5), \quad-2.265(\times 1), \\
& -3.486(\times 1), \quad-1.936(\times 5), \quad 0.386(\times 1), \quad 0(\times 9),
\end{aligned}
$$

showing two tachyon masses that violate the BF bound. The solution is unstable and does not contain flat directions. Computing the vector masses, they are given by

$$
m^{2} L^{2}=\frac{1}{7}(71-\sqrt{57} \pm \sqrt{1186+74 \sqrt{57}})(\times 3), \quad \frac{1}{7}(71-\sqrt{57})(\times 3), \quad 0(\times 3),
$$

then resembling those in (B.23) and also including the three $\mathrm{SO}(3)$ massless vectors. Looking at the gravitini mass matrix, one finds that this solution is non-supersymmetric. 
Vacuum 16. The flux background associated to this solution turns out to be quite involved

$$
\begin{array}{llll}
a_{0}=1.546 \lambda, & a_{1}=0.712 \lambda, & c_{0}=-0.542 \lambda, & b_{0}=-2.632 \lambda, \\
a_{2}=2.231 \lambda, & c_{3}^{\prime}=-0.112 \lambda, & b_{1}=1.626 \lambda, & d_{0}=-0.364 \lambda .
\end{array}
$$

Evaluated at this solution, the vacuum energy is $V_{0}=-0.294 \lambda^{2}$. The spectrum of scalar masses reads

$$
\begin{aligned}
m^{2} L^{2}= & 66.413(\times 1), \quad 18(\times 1), \quad 24.061(\times 1), \quad 21.246(\times 5), \\
& 12.341(\times 3), \quad 7.887(\times 1), \quad 4.122(\times 5), \quad-2.908(\times 1), \\
& 2.040(\times 5), \quad-1.857(\times 5), \quad 1.089(\times 1), \quad 0(\times 9),
\end{aligned}
$$

and contains one tachyon violating the BF bound and no flat directions. The set of vectors masses is given by

$$
m^{2} L^{2}=23.764(\times 3), \quad 18.900(\times 3), 3.920(\times 3), \quad 0(\times 3),
$$

showing the three massless vectors of the $\mathrm{SO}(3)$ symmetry. This solution is a non-supersymmetric critical point. We want to mention that there is a companion flux background obtained by exchanging $a_{1} \leftrightarrow-c_{0}, a_{2} \leftrightarrow-c_{3}^{\prime}$ and $b_{1} \leftrightarrow-d_{0}$ which produces the same mass spectra (B.28) and (B.29) and is also non-supersymmetric.

Vacuum 17. This is the last solution of the STU model we explore in this paper. The associated flux background reads

$$
\begin{aligned}
& a_{0}=-0.683 \lambda, \quad a_{1}=-0.837 \lambda, \quad c_{0}=-0.034 \lambda, \quad b_{0}=-0.252 \lambda, \\
& a_{2}=0.330 \lambda, \quad c_{3}^{\prime}=-0.757 \lambda, \quad b_{1}=0.073 \lambda, \quad d_{0}=-0.111 \lambda,
\end{aligned}
$$

and produces a vacuum energy $V_{0}=-0.065 \lambda^{2}$. The scalar masses are given by

$$
\begin{aligned}
m^{2} L^{2}= & 40.404(\times 5), \quad 18(\times 1), \quad 19.899(\times 1), \quad 16.974(\times 3), \\
& 15.001(\times 5), \quad 11.576(\times 5), \quad 7.810(\times 1), \quad 6.556(\times 1), \\
& -3.815(\times 1), \quad-2.102(\times 5), \quad 0.201(\times 1), \quad 0(\times 9) .
\end{aligned}
$$

There is one tachyon violating the BF bound and no flat directions. The spectrum of vector masses is

$$
m^{2} L^{2}=17.563(\times 3), \quad 15.305(\times 3), \quad 3.318(\times 3), \quad 0(\times 3),
$$

containing the three $\mathrm{SO}(3)$ massless vectors. Computing the gravitino mass matrix shows that this solution is non-supersymmetric. Finally, there is also a companion background if exchanging $a_{1} \leftrightarrow-c_{0}, a_{2} \leftrightarrow-c_{3}^{\prime}$ and $b_{1} \leftrightarrow-d_{0}$ which produces the same spectra (B.31) and (B.32) and is also non-supersymmetric.

Open Access. This article is distributed under the terms of the Creative Commons Attribution License (CC-BY 4.0), which permits any use, distribution and reproduction in any medium, provided the original author(s) and source are credited. 


\section{References}

[1] J. Scherk and J.H. Schwarz, How to Get Masses from Extra Dimensions, Nucl. Phys. B 153 (1979) 61 [INSPIRE].

[2] B. de Wit, H. Samtleben and M. Trigiante, The Maximal D=4 supergravities, JHEP 06 (2007) 049 [arXiv:0705.2101] [INSPIRE].

[3] J. Schon and M. Weidner, Gauged $N=4$ supergravities, JHEP 05 (2006) 034 [hep-th/0602024] [INSPIRE].

[4] J.-P. Derendinger, C. Kounnas, P.M. Petropoulos and F. Zwirner, Superpotentials in IIA compactifications with general fluxes, Nucl. Phys. B 715 (2005) 211 [hep-th/0411276] [INSPIRE].

[5] S. Kachru, R. Kallosh, A.D. Linde and S.P. Trivedi, De Sitter vacua in string theory, Phys. Rev. D 68 (2003) 046005 [hep-th/0301240] [INSPIRE].

[6] G. Villadoro and F. Zwirner, Beyond Twisted Tori, Phys. Lett. B 652 (2007) 118 [arXiv:0706.3049] [INSPIRE].

[7] D. Andriot and A. Betz, NS-branes, source corrected Bianchi identities and more on backgrounds with non-geometric fluxes, JHEP 07 (2014) 059 [arXiv:1402.5972] [INSPIRE].

[8] G. Dall'Agata, G. Villadoro and F. Zwirner, Type-IIA flux compactifications and $N=4$ gauged supergravities, JHEP 08 (2009) 018 [arXiv:0906.0370] [INSPIRE].

[9] G. Dibitetto, A. Guarino and D. Roest, Charting the landscape of $N=4$ flux compactifications, JHEP 03 (2011) 137 [arXiv:1102.0239] [INSPIRE].

[10] L.J. Romans, Massive N=2a Supergravity in Ten-Dimensions, Phys. Lett. B 169 (1986) 374 [INSPIRE].

[11] T. House and A. Micu, M-Theory compactifications on manifolds with $G_{2}$ structure, Class. Quant. Grav. 22 (2005) 1709 [hep-th/0412006] [InSPIRE].

[12] G. Dall'Agata and N. Prezas, Scherk-Schwarz reduction of M-theory on G2-manifolds with fluxes, JHEP 10 (2005) 103 [hep-th/0509052] [INSPIRE].

[13] C.M. Hull and R.A. Reid-Edwards, Flux compactifications of M-theory on twisted Tori, JHEP 10 (2006) 086 [hep-th/0603094] [INSPIRE].

[14] H. Looyestijn, E. Plauschinn and S. Vandoren, New potentials from Scherk-Schwarz reductions, JHEP 12 (2010) 016 [arXiv:1008.4286] [INSPIRE].

[15] L. Castellani and L.J. Romans, $N=3$ and $N=1$ Supersymmetry in a New Class of Solutions for $d=11$ Supergravity, Nucl. Phys. B 238 (1984) 683 [InSPIRE].

[16] L. Castellani, L.J. Romans and N.P. Warner, A classification of Compactifying Solutions for $d=11$ Supergravity, Nucl. Phys. B 241 (1984) 429 [INSPIRE].

[17] D. Cassani and P. Koerber, Tri-Sasakian consistent reduction, JHEP 01 (2012) 086 [arXiv:1110.5327] [INSPIRE].

[18] D. Cassani, P. Koerber and O. Varela, All homogeneous $N=2$ M-theory truncations with supersymmetric AdS4 vacua, JHEP 11 (2012) 173 [arXiv:1208.1262] [INSPIRE].

[19] G. Aldazabal, P.G. Camara, A. Font and L.E. Ibáñez, More dual fluxes and moduli fixing, JHEP 05 (2006) 070 [hep-th/0602089] [INSPIRE]. 
[20] C.M. Hull, Massive string theories from M-theory and F-theory, JHEP 11 (1998) 027 [hep-th/9811021] [INSPIRE].

[21] J. Shelton, W. Taylor and B. Wecht, Nongeometric flux compactifications, JHEP 10 (2005) 085 [hep-th/0508133] [INSPIRE].

[22] M. Graña, R. Minasian, H. Triendl and T. Van Riet, Quantization problem in Scherk-Schwarz compactifications, Phys. Rev. D 88 (2013) 085018 [arXiv:1305.0785] [INSPIRE].

[23] C. Beasley and E. Witten, A note on fluxes and superpotentials in M-theory compactifications on manifolds of $G_{2}$ holonomy, JHEP 07 (2002) 046 [hep-th/0203061] [INSPIRE].

[24] S. Gukov, Solitons, superpotentials and calibrations, Nucl. Phys. B 574 (2000) 169 [hep-th/9911011] [INSPIRE].

[25] B.S. Acharya and B.J. Spence, Flux, supersymmetry and M-theory on seven manifolds, hep-th/0007213 [INSPIRE].

[26] A. Font, A. Guarino and J.M. Moreno, Algebras and non-geometric flux vacua, JHEP 12 (2008) 050 [arXiv:0809.3748] [INSPIRE].

[27] T. Friedrich, On types of nonintegrable geometries, math/0205149 [INSPIRE].

[28] R.L. Bryant, Some remarks on $G_{2}$-structures, math/0305124 [INSPIRE].

[29] J. Held, BPS-like potential for compactifications of heterotic M-theory?, JHEP 10 (2011) 136 [arXiv: 1109.1974] [INSPIRE].

[30] G. Villadoro and F. Zwirner, $N=1$ effective potential from dual type-IIA D6/O6 orientifolds with general fluxes, JHEP 06 (2005) 047 [hep-th/0503169] [INSPIRE].

[31] G. Dibitetto, A. Guarino and D. Roest, Exceptional Flux Compactifications, JHEP 05 (2012) 056 [arXiv: 1202.0770] [INSPIRE].

[32] G. Dall'Agata and S. Ferrara, Gauged supergravity algebras from twisted tori compactifications with fluxes, Nucl. Phys. B 717 (2005) 223 [hep-th/0502066] [INSPIRE].

[33] G. Dall'Agata, R. D'Auria and S. Ferrara, Compactifications on twisted tori with fluxes and free differential algebras, Phys. Lett. B 619 (2005) 149 [hep-th/0503122] [INSPIRE].

[34] M. de Roo and P. Wagemans, Gauge Matter Coupling in $N=4$ Supergravity, Nucl. Phys. B 262 (1985) 644 [InSPIRE].

[35] G. Dibitetto, A. Guarino and D. Roest, How to halve maximal supergravity, JHEP 06 (2011) 030 [arXiv: 1104.3587] [INSPIRE].

[36] M.P. Hertzberg, S. Kachru, W. Taylor and M. Tegmark, Inflationary Constraints on Type IIA String Theory, JHEP 12 (2007) 095 [arXiv:0711.2512] [INSPIRE].

[37] E. Silverstein, Simple de Sitter Solutions, Phys. Rev. D 77 (2008) 106006 [arXiv: 0712.1196] [INSPIRE].

[38] S.S. Haque, G. Shiu, B. Underwood and T. Van Riet, Minimal simple de Sitter solutions, Phys. Rev. D 79 (2009) 086005 [arXiv:0810.5328] [INSPIRE].

[39] C. Caviezel et al., On the Cosmology of Type IIA Compactifications on SU(3)-structure Manifolds, JHEP 04 (2009) 010 [arXiv:0812.3551] [INSPIRE].

[40] U.H. Danielsson, S.S. Haque, G. Shiu and T. Van Riet, Towards Classical de Sitter Solutions in String Theory, JHEP 09 (2009) 114 [arXiv:0907.2041] [INSPIRE]. 
[41] G. Dibitetto, A. Guarino and D. Roest, Lobotomy of Flux Compactifications, JHEP 05 (2014) 067 [arXiv: 1402.4478] [INSPIRE].

[42] W. Decker, G.-M. Greuel, G. Pfister and H. Schönemann, Singular 3-1-5 - A computer algebra system for polynomial computations, http://www.singular.uni-kl.de, (2012).

[43] A. Borghese and D. Roest, Metastable supersymmetry breaking in extended supergravity, JHEP 05 (2011) 102 [arXiv: 1012.3736] [INSPIRE].

[44] P.G. Camara, A. Font and L.E. Ibáñez, Fluxes, moduli fixing and MSSM-like vacua in a simple IIA orientifold, JHEP 09 (2005) 013 [hep-th/0506066] [INSPIRE].

[45] G. Dibitetto, Private communication.

[46] P.G.O. Freund and M.A. Rubin, Dynamics of Dimensional Reduction, Phys. Lett. B 97 (1980) 233 [INSPIRE].

[47] A. Dabholkar and C. Hull, Duality twists, orbifolds and fluxes, JHEP 09 (2003) 054 [hep-th/0210209] [INSPIRE].

[48] A. Dabholkar and C. Hull, Generalised T-duality and non-geometric backgrounds, JHEP 05 (2006) 009 [hep-th/0512005] [INSPIRE].

[49] C. Condeescu, I. Florakis, C. Kounnas and D. Lüst, Gauged supergravities and non-geometric Q/R-fluxes from asymmetric orbifold CFT's, JHEP 10 (2013) 057 [arXiv: 1307.0999] [INSPIRE].

[50] P. Breitenlohner and D.Z. Freedman, Positive Energy in anti-de Sitter Backgrounds and Gauged Extended Supergravity, Phys. Lett. B 115 (1982) 197 [INSPIRE]. 